\title{
HST observations of the limb polarization of Titan
}

\author{
A. Bazzon ${ }^{1}$, H. M. Schmid ${ }^{1}$, and E. Buenzli ${ }^{2}$ \\ ${ }^{1}$ ETH Zurich, Institute of Astronomy, Wolfgang-Pauli-Str. 27, 8093 Zurich, Switzerland \\ e-mail: bazzon@astro.phys.ethz.ch \\ 2 Max-Planck-Institut für Astronomie, Königstuhl 17, 69117 Heidelberg, Germany
}

Received 27 November 2013 / Accepted 5 September 2014

\begin{abstract}
Context. Titan is an excellent test case for detailed studies of the scattering polarization from thick hazy atmospheres. Accurate scattering and polarization parameters have been provided by the in situ measurements of the Cassini-Huygens landing probe. For Earth-bound observations Titan can only be observed at a backscattering situation, where the disk-integrated polarization is close to zero. However, with resolved imaging polarimetry a second order polarization signal along the entire limb of Titan can be measured. Aims. We present the first limb polarization measurements of Titan, which are compared as a test to our limb polarization models. Methods. Previously unpublished imaging polarimetry from the HST archive is presented, which resolves the disk of Titan. We determine flux-weighted averages of the limb polarization and radial limb polarization profiles, and investigate the degradation and cancelation effects in the polarization signal due to the limited spatial resolution of our observations. Taking this into account we derive corrected values for the limb polarization in Titan. The results are compared with limb polarization models, using atmosphere and haze scattering parameters from the literature.

Results. In the wavelength bands between $250 \mathrm{~nm}$ and $2 \mu \mathrm{m}$ a strong limb polarization of about $2-7 \%$ is detected with a position angle perpendicular to the limb. The fractional polarization is highest around $1 \mu \mathrm{m}$. As a first approximation, the polarization seems to be equally strong along the entire limb. The comparison of our data with model calculations and the literature shows that the detected polarization is compatible with expectations from previous polarimetric observations taken with Voyager 2, Pioneer 11, and the Huygens probe.

Conclusions. Our results indicate that ground-based monitoring measurements of the limb-polarization of Titan could be useful for investigating local haze properties and the impact of short-term and seasonal variations of the hazy atmosphere of Titan. Planets with hazy atmospheres similar to Titan are particularly good candidates for detection with the polarimetric mode of the upcoming planet finder instrument at the VLT. Therefore, a good knowledge of the polarization properties of Titan is also important for the search and investigation of extra-solar planets.
\end{abstract}

Key words. polarization - planets and satellites: atmospheres - scattering - radiative transfer - instrumentation: polarimeters

\section{Introduction}

Solar light reflected from planets, moons, and smaller objects is polarized. This basic property of light reflection provides a powerful diagnostic tool for the remote investigation of the scattering particles in atmospheres and the reflecting surfaces of solar system bodies.

Polarization studies of Titan are particularly well suited to studying the scattering polarization from a hazy atmosphere. The hazy atmosphere of Titan produces a very strong polarization signal over a wide wavelength range. At quadrature phase $\alpha \approx 90^{\circ}$, the fractional polarization of Titan from the UV to the $R$ band is $p \approx 50 \%$, as measured by the Pioneer 11 (Tomasko $\&$ Smith 1982) and Voyager 2 (West et al. 1983) spacecrafts. Furthermore, thanks to the joint NASA-ESA Cassini-Huygens satellite mission, Titan's surface and atmospheric structure are known in great detail. In particular, the measurements made inside Titan's atmosphere, made available by the Huygens landing probe, have provided accurate scattering and polarization parameters for the haze particles (e.g., Brown et al. 2010; Tomasko et al. 2008).

For Earth-bound instruments the atmosphere of Titan can only be observed at very limited phase angles $\alpha \lesssim 5^{\circ}$, where the disk-integrated polarization is close to zero, and the polarimetric properties can only be investigated if disk-resolved imaging polarimetry is available. In this work we present previously unpublished imaging polarimetry from the HST archive which resolves the disk of Titan and clearly shows a strong limb polarization effect. Together with literature values for the albedo $A_{\mathrm{g}}$ and the quadrature polarization $p\left(90^{\circ}\right)$ of Titan, our limb polarization measurements can now be used to test polarization models for a haze scattering atmosphere, and we can make predictions for the detection and characterization of reflected light of extrasolar planets.

The limb polarization is a well-known second order scattering effect of reflecting atmospheres with predominantly Rayleigh-type scattering processes (e.g., van de Hulst 1980). In general, single backscattering with scattering angles $\sim 180^{\circ}$ would produce a very small polarization signal or no signal at all. Thus, the polarization measured at the limb arises from second order and also higher order scatterings by light that is scattered sideways, i.e., more or less parallel to the limb, and then scattered back to the observer. The polarization angle induced by Rayleigh scattering, i.e. single dipole-type scattering, is perpendicular to the propagation direction of the incoming photon. Hence, the position angle of polarization is perpendicular to the limb everywhere.

Over the last 20 years, Titan's thick and hazy atmosphere has been monitored and intensively studied by spectral HST observations, revealing strong local albedo variations mainly caused 
by seasonal migration of haze from one hemisphere to another (Lorenz et al. 2004, and references therein). The most prominent features are a varying north-south asymmetry, a dark polar hood that is most prominent in the UV, and a detached haze layer lying only $\sim 200 \mathrm{~km}$ above the optical limb of Titan (e.g., Lorenz et al. 2004, 2006). Observations of Titan from the ground, e.g., using the upcoming SPHERE instrument at the VLT (Beuzit et al. 2008), consisting of state-of-the-art imagers and polarimeters, will have the advantage of a much higher spatial resolution and a broader wavelength coverage. Therefore, a consistent monitoring program of Titan's atmosphere from the ground could potentially be useful for investigating the temporal changes in the polarization structure along the limb, and for constraining local haze properties of Titan.

After the description of the observational data and the basic data reduction in Sects. 2 and 3 respectively, we discuss the intensity images in Sect. 4 and compare them with the literature. In Sect. 5 we derive the Stokes images for all our wavelength bands, which are then converted into radial limb polarization images in Sect. 6. There, we discuss the radial polarization profiles and the advantage of disk-integrated radial polarization measurements, and we explain how we correct our images for the polarization degradation caused by a PSF smearing effect. In Sect. 7 we describe our radiative transfer model for Titan, and we compare the model with our limb polarization results and literature values for the geometric albedo $A_{\mathrm{g}}$ and the quadrature polarization $p\left(90^{\circ}\right)$ of Titan. The last section gives a summary and discusses the prospects for a polarimetric monitoring program of Titan and for the detection and investigation of extra-solar haze planets.

\section{Observations}

We reduced and analyzed imaging polarimetry of Titan from the HST archive $^{1}$ for which only the intensity images have been published (Lorenz et al. 2006) but not the polarization data. The data were recorded with the ACS HRC and the NICMOS instruments in seven filters covering wavelengths $0.25 \mu \mathrm{m}-2 \mu \mathrm{m}$. Polarimetry is achieved with three subsequent measurements, using three polarizers with different orientations. Titan was observed in 2002 during two visits on November 27 and December 2, i.e., shortly after southern summer solstice that occurred in late October 2002, and with the north pole of Titan on the hidden hemisphere. Table 1 gives an overview of the observational parameters, the used instruments and corresponding filters, the total exposure times, and the plate scales.

For broad-band observations the nominal filter wavelength may differ significantly from the average wavelength of the photons registered in the polarization map. Similarly the evaluation of the reflected-flux weighted albedo $A_{\mathrm{g}, \text { eff }}$ for a given filter considers a weighting with the effective spectral distribution of the registered photons, taking into account the wavelength dependence of the instrument, the solar photon spectrum, and the albedo of Titan.

Figure 1 illustrates the spectral dependence of the full disk albedo of Titan from the mid-UV to the near-IR (McGrath et al. 1998 for the UV, Karkoschka 1998 for the visual, Negrão et al. 2006 for the near-IR) assuming Titan's optical radius varying with $\lambda$ according to the Toon et al. (1992) radius $R_{\mathrm{T}}$. The solar irradiance photon spectrum derived from Thuillier et al. (2004) is given by the dashed line. The normalized instrument efficiencies in the different HST filters and the calculated spectral distribution of the registered photons are also indicated. We use the

\footnotetext{
1 HST proposal ID 9385.
}

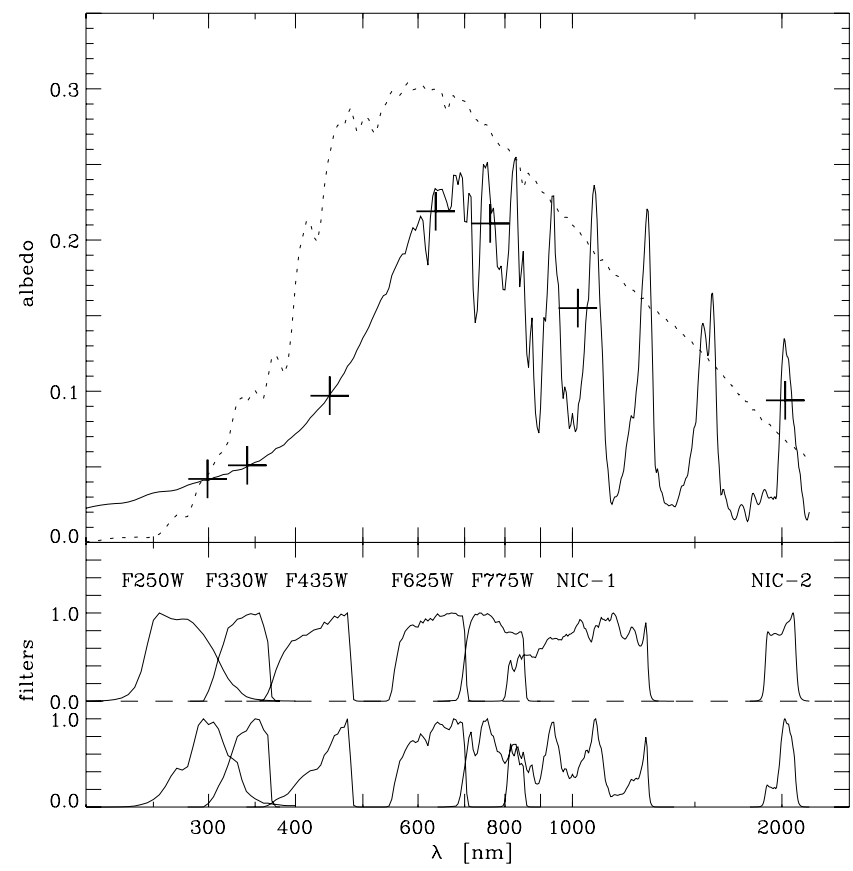

Fig. 1. Upper panel: geometric albedo of Titan (full line) and effective albedos $A_{\mathrm{g} \text {,eff }}$ and wavelengths $\lambda_{\mathrm{eff}}$ for the HST filter polarimetry (crosses). The dashed line illustrates the solar spectrum. Lower panel: normalized filter efficiency curves and the normalized wavelength distribution of the registered photons.

median wavelengths of the individual photon distributions as effective filter wavelengths $\lambda_{\text {eff }}$, and the reflected-flux weighted values for the effective albedos $A_{\mathrm{g}, \text { eff }}$ for each filter. The corresponding values are indicated in Fig. 1 and Table 3 lists the derived values and $R_{\mathrm{T}}\left(\lambda_{\text {eff }}\right)$.

We note that when looking specifically at the limb the methane bands are weaker and center-to-limb differences in the albedo spectrum are present (e.g., Smith et al. 1996; Lorenz et al. 2004, 2006). However, we mainly focus on the derivation of disk-integrated albedo and limb polarization values (Sect. 6.3).

The effective filter wavelengths $\lambda_{\mathrm{eff}}$ are shifted to longer wavelength for the UV/blue filters because of the steep photon spectrum. The effective albedos $A_{\mathrm{g}, \text { eff }}$ are relevant for the filters covering spectral regions with strong $\mathrm{CH}_{4}$ absorption bands. They are about $20 \%$ higher for the NICl and $40 \%$ higher for the NIC2 passbands than the simple mean. For the $F 250 \mathrm{~W}$ and the $F 330 W$ filters the difference is $<5 \%$, and for the other filters the difference is $\sim 10 \%$ or less (steep flux gradient combined with systematic albedo gradient).

\section{Basic data reduction}

The data provided by the HST data reduction pipeline are already corrected for bias, dark, flatfield, and image distortion. However, in the case of the ACS data the pipeline drizzled, combined images showed a strange stripe pattern which was caused by incorrectly set bits in the data quality mask that are used for identifying pixels flagged as cosmic rays by MultiDrizzle ${ }^{2}$. Using the instructions provided by the stsdas $^{3}$ helpdesk we reset the dq_bits and re-run multidrizzle using the standard settings.

Both the ACS and the NICMOS instrument contain sets of three linear polarizer filters with their relative polarization

\footnotetext{
2 The MultiDrizzle Handbook, Chap. 5.4.6.3 "Final Products".

3 Space Telescope Science Data Analysis System.
} 
Table 1. Observational parameters and summary of the HST polarimetry used in this work.

\begin{tabular}{|c|c|c|}
\hline & ACS HRC & NICMOS 1 and 2 \\
\hline \multicolumn{3}{|l|}{ Obs. parameters ${ }^{a, b}$} \\
\hline Date & 2002-12-02 & $2002-11-27$ \\
\hline Diameter & $0.88^{\prime \prime}$ & $0.88^{\prime \prime}$ \\
\hline Phase angle & $1.8^{\circ}$ & $2.4^{\circ}$ \\
\hline NP distance & $-0.39^{\prime \prime}$ & $-0.39^{\prime \prime}$ \\
\hline Angle NP-N $\mathrm{N}_{\text {cel }}$ & $-5.3^{\circ}$ & $-5.3^{\circ}$ \\
\hline \multicolumn{3}{|l|}{ Exposures } \\
\hline F250W & $3 \times 4 \times 365 s$ & - \\
\hline F330W & $3 \times 4 \times 200 \mathrm{~s}$ & - \\
\hline$F 435 W$ & $3 \times 4 \times 45 s$ & - \\
\hline F625W & $3 \times 2 \times 12 s$ & _ \\
\hline F775W & $3 \times 2 \times 9 \mathrm{~s}$ & - \\
\hline POL S & - & $3 \times 2 \times 20 s$ \\
\hline POL L & _- & $3 \times 2 \times 12 \mathrm{~s}$ \\
\hline Plate scale & $0.025^{\prime \prime} / \mathrm{pix}$ & $0.043^{\prime \prime} / \mathrm{pix} \& 0.075^{\prime \prime} / \mathrm{pix}$ \\
\hline
\end{tabular}

Notes. The southern summer solstice of Titan occurred in late October 2002. NP distance gives the angular distance of the north pole from the center of the disk, whereas the negative distance indicates that the north pole is on the hidden hemisphere. ${ }^{(a)}$ US Naval Observatory \& Royal Greenwich Observatory (2000). ${ }^{(b)}$ http://ssd.jpl.nasa. gov/?horizons.

directions oriented according to $0^{\circ}, 60^{\circ}, 120^{\circ}$ and $0^{\circ}, 120^{\circ}, 240^{\circ}$ respectively. In the first data reduction step the basic pipeline processed images were cut out and aligned to subpixel accuracy of \pm 0.1 pixel. Then the images corresponding to the Stokes parameters $I, Q$, and $U$ were calculated and corrected for instrumental polarization.

\subsection{Calculation of Stokes parameters for ACS}

In case of ACS the Stokes parameters are calculated according to:

$$
\begin{aligned}
I & =\left(\frac{2}{3}\right)\left[i_{0} \cdot c_{0}(\lambda)+i_{60} \cdot c_{60}(\lambda)+i_{120} \cdot c_{120}(\lambda)\right], \\
Q & =\left(\frac{2}{3}\right)\left[2 i_{0} \cdot c_{0}(\lambda)-i_{60} \cdot c_{60}(\lambda)-i_{120} \cdot c_{120}(\lambda)\right]\left(\frac{T_{\|}+T_{\perp}}{T_{\|}-T_{\perp}}\right), \\
U & =\left(\frac{2}{\sqrt{3}}\right)\left[i_{60} \cdot c_{60}(\lambda)-i_{120} \cdot c_{120}(\lambda)\right]\left(\frac{T_{\|}+T_{\perp}}{T_{\|}-T_{\perp}}\right),
\end{aligned}
$$

whereas $i_{*}$ indicate the intensity images corresponding to the three polarizer orientations, $c_{*}$ are corresponding correction factors calibrating the polarization zero-point, and the $T_{*}$ parameters correct for polarization cross-talks caused by leakages of the polarizing filters. The calibration parameters are given in the HST calibration handbook (Fig. 5.3, Table 6.3) which we summarize in Table 2. According to the handbook for the ACS camera the residual instrumental polarization uncertainty should be at the one-part-in-ten level for highly polarized sources and at the $1 \%$ level for weakly polarized targets.

For the $F 250 \mathrm{~W}$ filter no calibration parameters are provided by the HST handbook. However, because of symmetry reasons one can assume that to first order the fractional polarization at the center of the apparent disk of Titan should be zero:

$(Q / I)_{\text {center }} \approx(U / I)_{\text {center }} \approx 0$.

Therefore, by using extrapolated values for $c_{*}$ as starting points, and by minimizing the fractional polarization around the center of Titan, we determined estimates for the $F 250 \mathrm{~W}$ calibration
Table 2. Calibration parameters for ACS polarimetry.

\begin{tabular}{cccccc}
\hline \hline Spectral filter & $c_{0}$ & $c_{60}$ & $c_{120}$ & $T_{\|}$ & $T_{\perp}$ \\
\hline$F 250 W^{* *}$ & 1.840 & 1.625 & 1.801 & 0.28 & 0.02 \\
$F 330 W$ & 1.7302 & 1.5302 & 1.6451 & 0.475 & 0.05 \\
$F 435 W$ & 1.6378 & 1.4113 & 1.4762 & 0.525 & 0.02 \\
$F 625 W^{*}$ & 1.0443 & 0.9788 & 0.9797 & 0.500 & 0.00 \\
$F 775 W$ & 1.0867 & 1.0106 & 1.0442 & 0.650 & 0.00 \\
\hline
\end{tabular}

Notes. ${ }^{(*)}$ Calibration not scaled for Stokes I. According to HST handbook there is also some evidence of a polarization pathology. ${ }^{(* *)}$ No calibration parameters available. See text for the derivation of these values.

parameters indicated in Table 2. Especially for the integrated radial polarization described in Sect. 6 these calibration parameters produce very reasonable results which are consistent with the other wavelengths.

\subsection{Calculation of Stokes parameters for NICMOS}

In case of NICMOS the HST handbook provides the user with two coefficient matrices $M_{1 ; 2}$ to calculate the Stokes parameters of $\mathrm{NIC1}$ and NIC2 respectively:

$$
\left(\begin{array}{c}
I \\
Q \\
U
\end{array}\right)=M_{1 ; 2}^{-1}\left(\begin{array}{c}
i_{0} \\
i_{120} \\
i_{240}
\end{array}\right)
$$

with the matrices

$$
M_{N I C l}=\left(\begin{array}{ccc}
0.3936 & 0.3820 & 0.0189 \\
0.3959 & -0.1118 & -0.1463 \\
0.3902 & -0.2768 & 0.1150
\end{array}\right)
$$

$$
M_{\text {NIC2 }}=\left(\begin{array}{ccc}
0.5094 & 0.3550 & 0.1131 \\
0.5139 & -0.0403 & -0.3206 \\
0.5159 & -0.3262 & 0.3111
\end{array}\right) .
$$

For NICl the coefficient matrix calibration is not perfect and a residual instrumental polarization at a level of $p_{\text {inst. }} \approx 1.2-1.5 \%$ was reported (see Batcheldor et al. 2009). Furthermore, for bright targets ghost images are present in two NIC1 polarization filters $\left(i_{0^{\circ}}, i_{240^{\circ}}\right)$. In case of NIC 2 the instrumental effects are very well calibrated, and uncertainties as low as $p_{\text {inst. }} \approx 0.2 \%$ should be achievable with bright objects. Both for NICl and $N I C 2$, this is in good agreement with our findings for the Titan polarization in the disk center in Sect. 5.

\section{Intensity images}

The used HST-dataset was mainly taken to study seasonal effects of the stratospheric haze on Titan, and an analysis of the spectro-photometric data is given by Lorenz et al. (2004, 2006). In particular, they describe and explain in detail the varying dark polar hood and the north-south asymmetry measured in different narrow-band filters.

The dark polar hood was first seen in 1980 by Voyager 1 around the north, and disappeared from the south pole in 2002-2003 (Lorenz et al. 2006). Most probably, the polar hood is associated to a downwelling during the long polar night, redistributing haze from the summer hemisphere towards the winter pole (Rannou et al. 2002; Lorenz et al. 2006). This process then also creates the detached haze layer as the haze is horizontally drawn from beneath the formation zone. 


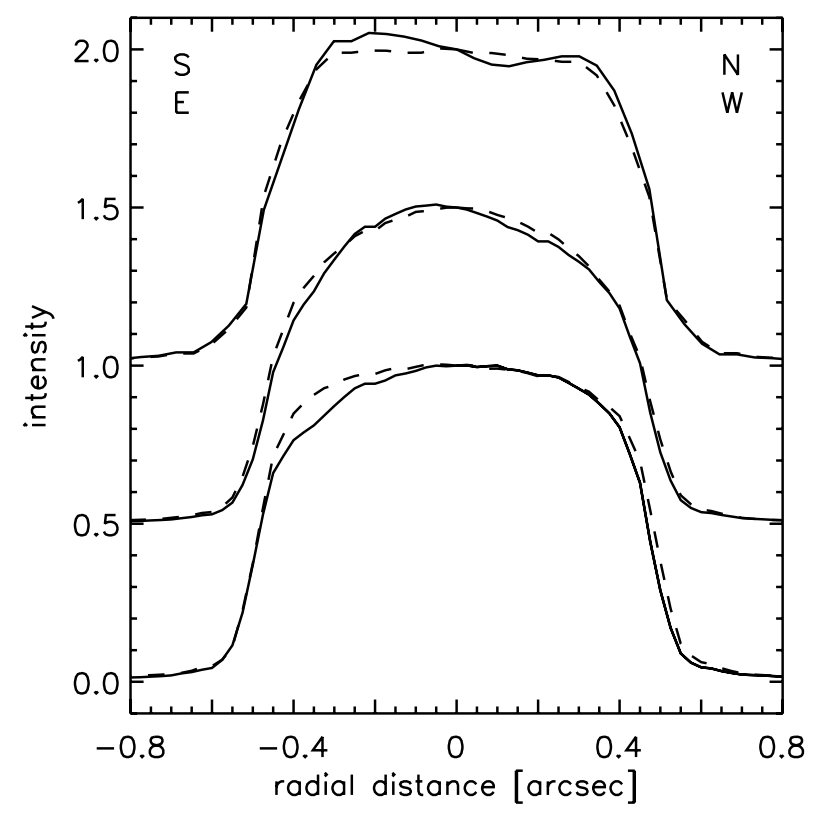

Fig. 2. Intensity cuts/profiles through the disk center for the $F 250 \mathrm{~W}$ band (bottom), the F435W band (middle), and the NICl band (top). The solid line indicates the north-south profile through the planetary poles, and the dashed line is the east-west profile perpendicular to the polar axis respectively.

Lorenz et al. (2004) find that in the narrow-band filters the north-south asymmetry is reversed between the blue $(439 \mathrm{~nm})$ and the red $(889 \mathrm{~nm})$, and almost absent at $619 \mathrm{~nm}$. In the blue Titan is brighter in the south than in the north and vice versa in the red methane bands. In the near-IR, the variation of the asymmetry with wavelength is dramatic, and different narrow-band filters may see different reversions of the north-south asymmetry as they are probing different altitude regions.

The north-south asymmetry is also visible in our broad-band images ranging from $0.3-2 \mu \mathrm{m}$ of the same HST visit as the narrow-band data of Lorenz et al. (2004), and it is in qualitative agreement with the spectro-photometric analysis by Lorenz et al. (2004, 2006). Figure 2 shows N-S and E-W profiles of Titan for the $F 330 \mathrm{~W}$ band, the $F 435 \mathrm{~W}$ band, and the NICl band. Intensity images for the bands $F 330 \mathrm{~W}, \mathrm{~F} 435 \mathrm{~W}, \mathrm{~F} 625 \mathrm{~W}, \mathrm{NIC1}$, and NIC2 are given in the left panel of Fig. 5. The nominal optical radius of Titan, the south pole, and the equator are also indicated. The images are normalized such that

$\int_{0}^{R_{\mathrm{int}}} \int_{0}^{2 \pi} \operatorname{Ir} \mathrm{d} r \mathrm{~d} \phi=A_{\mathrm{g}, \mathrm{eff}} \cdot \pi R_{\mathrm{int}}^{2}$,

where $A_{\mathrm{g}, \text { eff }}$ indicates the effective albedo given in Table 3 and Fig. 1 , and $R_{\text {int }}=0.75^{\prime \prime}$ is the integration radius, which is greater than the nominal radius of Titan $R_{\text {Titan }}=0.44^{\prime \prime}$.

\section{Stokes $Q$ and $U$ images for Titan}

In Fig. 3 Stokes $Q$ and $U$ images in the $F 775 W$ band are shown. The same highly symmetric quadrant pattern is present in all of our data. This butterfly pattern is real and it is practically impossible to artificially create such a pattern by misalignments of the three polarization images $i_{*}$ or other spurious effects. The gray scale is normalized to the central intensity $I_{\text {center }}$ of the planetary disk and goes from $-0.02 I_{\text {center }}$ (black) to $+0.02 I_{\text {center }}$ (white). At the center $Q$ and $U$ are essentially zero $(Q / I, U / I \lesssim \pm 0.2 \%)$.

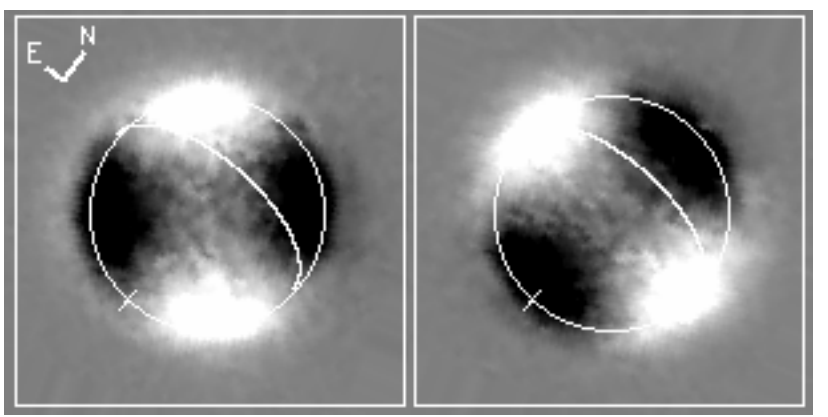

Fig. 3. Stokes $Q$ (left) and $U$ (right) images in the $F 775 \mathrm{~W}$ band. The south pole and the equator are indicated. The gray scale is normalized to the central intensity of Stokes $I$ by $\pm 0.02 I_{\text {center }}$.

The butterfly polarization pattern is typical for a centrosymmetric scattering geometry. For example, the Rayleighscattering atmospheres of Uranus and Neptune show this pattern of radial limb polarization (Schmid et al. 2006b). For all our data the pattern is highly symmetric, indicating that the limb polarization has similar strength along the entire limb of Titan. The strength of the limb polarization increases with wavelength until it peaks in the $1 \mu \mathrm{m}$ band measurement after which it decreases again.

We do not see a significant imprint of the north-south asymmetry of Titan (Sect. 4) in the polarization images. To study the polarization along the north-south (and east-west) direction the polarization pattern was aligned with respect to the polar axis of Titan by

$Q_{\mathrm{NS}}=Q \cos \left(2 \theta_{\mathrm{NP}}\right)-U \sin \left(2 \theta_{\mathrm{NP}}\right)$,

where $\theta_{\mathrm{NP}}$ is the angle between the polarizer reference axis and the polar axis of Titan.

For all filters we calculate disk-integrated Stokes fluxes $\Sigma I$, $\Sigma Q$, and $\Sigma U$ by summing up all counts within the integration radius $R_{\text {int }}=0.75^{\prime \prime}$ from the apparent disk center of the $I, Q$, and $U$ images respectively. $R_{\text {int }}$ includes the halo of the planet which is greater than the nominal limb at $R_{\text {Titan }}=0.44^{\prime \prime}$. We then calculate disk-integrated fractional polarization parameters for Stokes $Q$ (and similarly $U$ ):

$\langle Q / I\rangle_{\mathrm{m}}\left(R_{\mathrm{int}}\right)=\Sigma Q / \Sigma I$

$\langle Q / I\rangle_{\mathrm{m}}$ and $\langle U / I\rangle_{\mathrm{m}}$ are equivalent to a measurement with aperture polarimetry, where the aperture is larger than the planet. The results for all filters are given in Table 3 . The disk-integrated polarization of Titan is essentially zero $(p<0.2 \%)$ for all filters in agreement with Veverka (1973) and Zellner (1973), except for F330W and for NICl; these results are not real and can be explained by instrumental effects.

As explained in Sect. 3.2 NIC1 is not well calibrated for instrumental polarization and between $p_{\text {inst. }} \approx 1.2 \%-1.5 \%$ residual polarization is expected. We are measuring $\langle Q / I\rangle_{\mathrm{m}}=1.5 \%$ despite the expectation that Titan has zero net polarization. The same instrument offset is also seen at the center of the disk which should be zero because of symmetry reasons. Similarly for the $F 330 \mathrm{~W}$ measurement the center of the Stokes $Q$ and $U$ images is not zero indicating residual instrumental polarization at the level of $p_{\text {inst. }} \approx 0.8 \%$ in $Q$ and $p_{\text {inst. }} \approx 0.2 \%$ in $U$ respectively.

The absence of a net polarization in the disk averages indicates that the limb polarization has a similar strength along the entire limb for all observed bands. We note that the diskintegrated parameters $\langle Q / I\rangle_{\mathrm{m}}$ and $\langle U / I\rangle_{\mathrm{m}}$ can hardly be affected 
Table 3. Polarization results for Titan.

\begin{tabular}{|c|c|c|c|c|c|c|c|c|c|c|c|c|}
\hline \multirow[b]{2}{*}{ Filter } & \multirow[b]{2}{*}{$\begin{array}{c}\lambda_{\mathrm{c}} \\
{[\mathrm{nm}]}\end{array}$} & \multirow[b]{2}{*}{$\begin{array}{c}\lambda_{\mathrm{eff}} \\
{[\mathrm{nm}]}\end{array}$} & \multirow[b]{2}{*}{$A_{\mathrm{g}, \mathrm{eff}}$} & \multirow[b]{2}{*}{$\begin{array}{c}R_{\mathrm{T}} \\
{[\mathrm{km}]}\end{array}$} & \multicolumn{2}{|c|}{ Integrated polarization } & \multicolumn{4}{|c|}{ Integrated radial polarization } & \multicolumn{2}{|c|}{ Max. radial pol. } \\
\hline & & & & & $\begin{array}{c}\langle Q / I\rangle_{\mathrm{m}} \\
{[\%]}\end{array}$ & $\begin{array}{c}\langle U / I\rangle_{\mathrm{m}} \\
{[\%]}\end{array}$ & $\begin{array}{c}\left\langle Q_{\mathrm{r}} / I\right\rangle_{\mathrm{m}} \\
{[\%]}\end{array}$ & $\begin{array}{c}\left\langle U_{\mathrm{r}} / I\right\rangle_{\mathrm{m}} \\
{[\%]}\end{array}$ & $C_{\mathrm{PSF}}$ & $\begin{array}{c}\left\langle Q_{\mathrm{r}} / I\right\rangle \\
{[\%]}\end{array}$ & $\begin{array}{c}\left(Q_{\mathrm{r}} / I\right)_{\mathrm{m}}^{\max } \\
{[\%]}\end{array}$ & $\begin{array}{c}\left(Q_{\mathrm{r}} / I\right)^{\max } \\
{[\%]}\end{array}$ \\
\hline$\overline{F 250 W}$ & 273 & 299 & 0.042 & 2901 & 0.13 & 0.00 & 0.99 & 0.03 & 0.84 & 1.18 & 1.82 & 4.18 \\
\hline F330W & 336 & 341 & 0.051 & 2892 & -0.81 & -0.36 & 1.10 & -0.05 & 0.85 & 1.30 & 2.37 & 4.40 \\
\hline$F 435 W$ & 432 & 448 & 0.097 & 2871 & 0.06 & 0.08 & 1.71 & -0.09 & 0.86 & 1.98 & 3.44 & 5.31 \\
\hline F625W & 632 & 636 & 0.219 & 2835 & 0.00 & 0.19 & 2.51 & -0.09 & 0.85 & 2.95 & 4.93 & 6.86 \\
\hline$F 775 W$ & 768 & 762 & 0.211 & 2813 & 0.09 & 0.03 & 2.97 & -0.14 & 0.82 & 3.63 & 5.19 & 8.70 \\
\hline NICl & 1071 & 1018 & 0.155 & 2771 & -1.49 & -1.40 & 4.03 & 0.04 & 0.74 & 5.45 & 6.90 & 10.49 \\
\hline $\mathrm{NIC} 2$ & 2002 & 2022 & 0.094 & 2651 & -0.30 & -0.09 & 1.99 & 0.00 & 0.58 & 3.42 & 3.44 & _* \\
\hline
\end{tabular}

Notes. The columns give the filter, the central wavelength $\lambda_{\mathrm{c}}$, the effective wavelength $\lambda_{\mathrm{eff}}$, the effective albedo $A_{\mathrm{g} \text {,eff }}$ for the photons registered in the broad-band filters, and the Toon et al. (1992) radius $R_{\mathrm{T}}$ for $\lambda_{\mathrm{eff}} .\langle Q / I\rangle_{\mathrm{m}},\langle U / I\rangle_{\mathrm{m}},\left\langle Q_{\mathrm{r}} / I\right\rangle_{\mathrm{m}}$, and $\left\langle U_{\mathrm{r}} / I\right\rangle_{\mathrm{m}}$ are the measured disk-integrated polarization parameters. The parameter $C_{\mathrm{PSF}}$ describes the degradation of the polarization measurement due to the PSF smearing effect (Sect. 6.4), and the corrected radial polarization value is given by $\left\langle Q_{\mathrm{r}} / I\right\rangle=\left\langle Q_{\mathrm{r}} / I\right\rangle_{\mathrm{m}} / C_{\mathrm{PSF}} .\left(Q_{\mathrm{r}} / I\right)_{\mathrm{m}}^{\mathrm{max}}$ is the measured maximum radial polarization, whereas $\left(Q_{\mathrm{r}} / I\right)^{\mathrm{max}}$ is the modeled value for infinite resolution. The statistical $1 \sigma$ error for the disk-integrated polarization is estimated to be $\Delta p \leq \pm 0.1 \%$ and $\Delta C_{\mathrm{PSF}}= \pm 0.01$. In addition, a systematic uncertainty of $(\Delta p)_{\text {syst. }} \approx \pm 0.2 \%$ is estimated. ${ }^{(*)}$ wavelength range of model: $200 \mathrm{~nm}<\lambda<1600 \mathrm{~nm}$.

by inaccuracies in the image centering procedure or other spurious effects due to the data reduction. The statistical $1 \sigma$ measuring error is $\Delta p<0.1 \%$ as estimated for the measuring error of the integrated radial polarization $\left\langle U_{\mathrm{r}} / I\right\rangle_{\mathrm{m}}$ in Sect. 6 which is independent of any residual instrumental offset.

\section{The radial polarization}

The polarization flux of an object is given by $p \times I=\sqrt{Q^{2}+U^{2}}$. However, because of the squares in this formula, large systematic bias errors are introduced if the absolute value of one or both measured signals $|Q|$ and $|U|$ is not significantly higher than the measuring noise $\Delta Q$ and $\Delta U$. In our Titan data there is $\Delta Q \approx|Q|$ and $\Delta U \approx|U|$ in the middle of the planetary disk, and between positive and negative quadrants in the butterfly pattern. Therefore one should not use the polarized flux $p \times I$ or the normalized polarization $p$ as measuring parameter. We adopt radial Stokes parameters, which are particularly well-suited for characterizing centro-symmetric polarization patterns of planets (e.g., Schmid et al. 2006b).

The radial Stokes parameters $Q_{\mathrm{r}}$ and $U_{\mathrm{r}}$ describe the polarization in radial and tangential direction on the disk of Titan. They are given by

$Q_{\mathrm{r}}=+Q \cos 2 \phi+U \sin 2 \phi$
$U_{\mathrm{r}}=-Q \sin 2 \phi+U \cos 2 \phi$,

where $\phi$ is the polar angle of a given position $(x, y)$ on the apparent planetary disk (disk center $\left.\left(x_{0}, y_{0}\right)\right)$ with respect to the polarizer reference direction:

$\phi=\arctan \frac{x-x_{0}}{y-y_{0}}$.

$Q_{\mathrm{r}}>0$ is equivalent to a radial polarization or a polarization perpendicular to the limb, while $Q_{\mathrm{r}}<0$ indicates a tangential polarization component. $U_{\mathrm{r}}$ describes the polarization in the directions $\pm 45^{\circ}$ with respect to the radial direction.

\subsection{Radial stokes $Q_{r}$ and $U_{r}$ images for Titan}

For all observed bands we calculated radial polarization images $Q_{\mathrm{r}}$ and $U_{\mathrm{r}}$. In case of the $F 330 \mathrm{~W}$ and NIC1 observations the Stokes $Q$ and $U$ images were first corrected for residual instrumental polarization (see Sect. 5), derived from the disk center

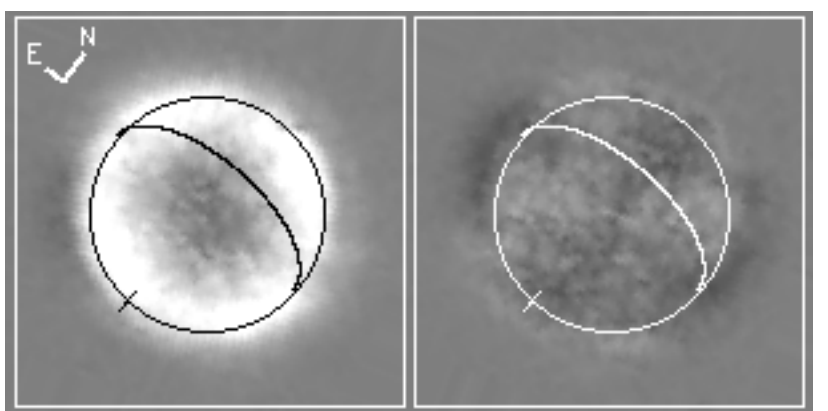

Fig. 4. Radial Stokes $Q_{\mathrm{r}}(l e f t)$ and $U_{\mathrm{r}}(r i g h t)$ images in the $F 775 \mathrm{~W}$ band. The south pole and the equator are indicated. The gray scale is normalized to the central intensity of Stokes $I$ by $\pm 0.02 I_{\text {center }}$.

which is expected to be unpolarized. Figure 4 shows $Q_{\mathrm{r}}$ and $U_{\mathrm{r}}$ for Titan in the $F 775 \mathrm{~W}$ filter. In all of our data the limb polarization is clearly visible as a bright ring with positive $Q_{\mathrm{r}}$ polarization and essentially zero $U_{\mathrm{r}}$ polarization. Except for the $F 250 \mathrm{~W}$ filter, the level of the $U_{\mathrm{r}}$ polarization is typically about 10 times lower than the positive $Q_{\mathrm{r}}$ signal along the limb. For the $F 250 \mathrm{~W}$ filter the $U_{\mathrm{r}}$ polarization is about 3 times lower than $Q_{\mathrm{r}}$. Thus $Q_{\mathrm{r}}$ dominates in all filters. In Fig. 5 we show images of $Q_{\mathrm{r}}$ as well as corresponding Stokes $I$ for the rest of our data. The gray scale of the radial polarization images in Figs. 4 and 5 is scaled to the central intensity $I_{\text {center }}$ by $\pm 0.02 \cdot I_{\text {center }}$, and the intensity images are normalized to the effective albedo $A_{\mathrm{g}, \text { eff }}$ according to Eq. (1).

\subsection{Polarization as function of radius}

The observed polarization of Titan is essentially centrosymmetric, and contrary to the strong north-south albedo asymmetry, the corresponding imprint in the radial polarization flux is either absent or much weaker. In the fractional radial polarization images $Q_{\mathrm{r}} / I$ we see marginal north-south differences with higher polarization in the north for the bands shorter than $1 \mu \mathrm{m}$, the opposite effect in the NICl band, and about equal polarization for the NIC2 band. However, these results are not significant and on the order of our systematic uncertainties $\Delta p / p \approx$ $0.05-0.1$.

In the infrared there is a similar weak indication for an east-west asymmetry of $Q_{\mathrm{r}} / I$, whereas for the NICl band the 

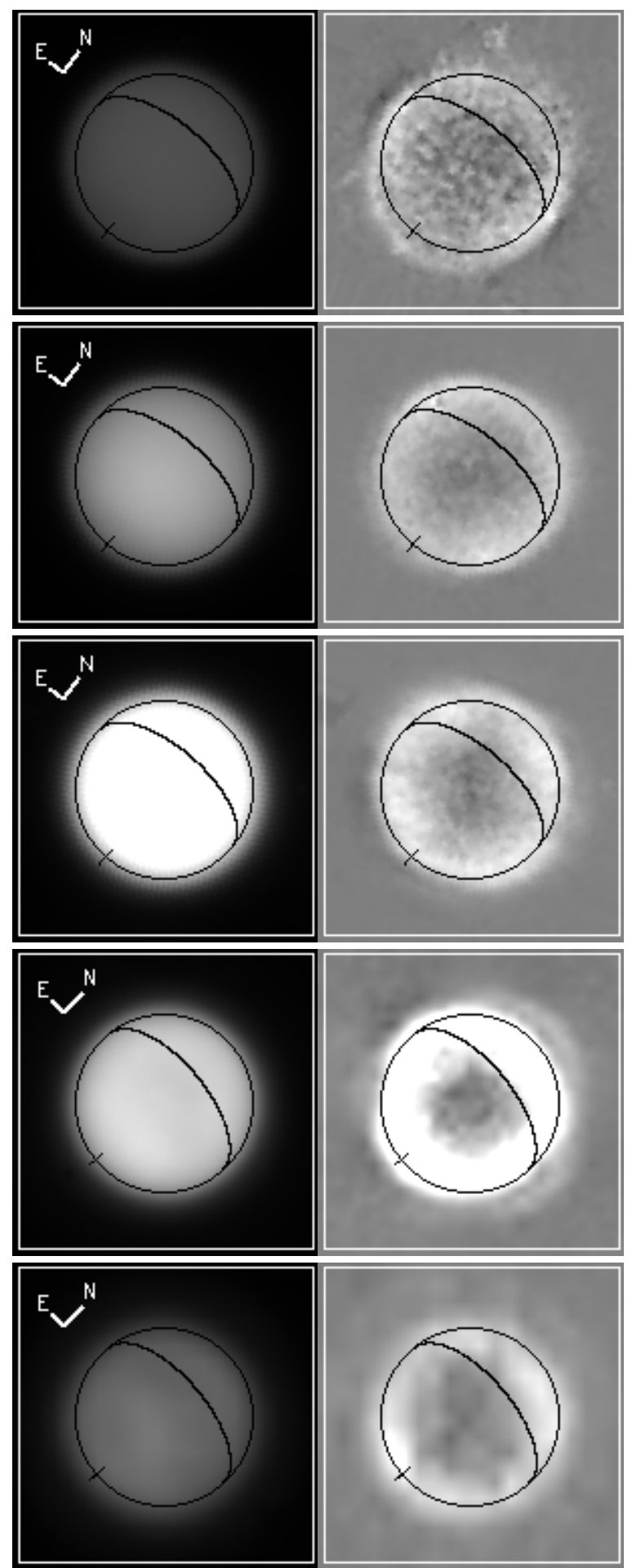

Fig. 5. Intensity (left) and radial polarization images $Q_{\mathrm{r}}($ right $)$ in the F330W, F435W, F625W, NICl, and NIC2 band (top to bottom). The intensity images are normalized to $\int_{0}^{R} \int_{0}^{2 \pi} I r \mathrm{~d} r \mathrm{~d} \phi=A_{\mathrm{g}, \mathrm{eff}} \cdot \pi R^{2}$ and the gray scale of the polarization images is scaled to $\pm 2 \%$ of the central intensity $I_{\text {center }}$.

polarization seems to be higher in the east, which is inverted for the NIC2 band. It also seems that for wavelengths shorter than $1 \mu \mathrm{m}$ the maximum polarization is higher at the eastern and western limbs than in the north and south, whereas for the NICl band the polarization in east-west and north-south is of about equal strength, and for NIC 2 the polarization is higher at

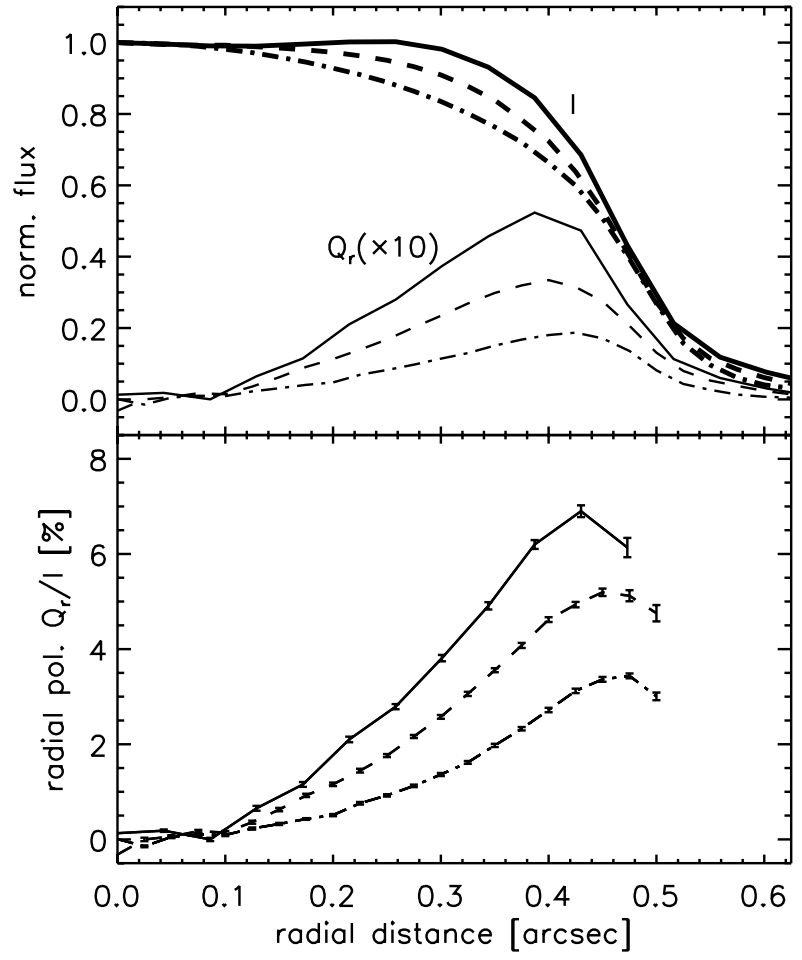

Fig. 6. Titan radial profiles for intensity $I$ (upper panel, thick lines) and radial polarization $Q_{\mathrm{r}}$ (upper panel, thin lines) in the $F 435 \mathrm{~W}$ (dash-dot), $F 775 W$ (dash) and $N I C 1$ (solid) filters. Both $I$ and $Q_{\mathrm{r}}$ are normalized to the peak flux at $r=0$. The lower panel shows the corresponding normalized radial polarization $Q_{\mathrm{r}} / I$.

the poles. However, the quality of our data is not good enough to draw firm conclusions, and at least part of the polarization differences mentioned above could be due to systematics in the data reduction, e.g., slight misalignments of corresponding polarization images coupled with strong intensity gradients at the limb.

The detection of structure in the limb polarization would be very interesting for investigating local haze properties, e.g., such as particle size differences of the photochemical haze between the morning and the evening limb of Titan. Observations with higher polarimetric sensitivity and higher spatial resolution are required for such studies.

For the moment, assuming a rotational symmetry for the polarization structure seems to be a reasonable first approximation, and we construct rotationally averaged, radial profiles for the polarization, the normalized polarization, and the intensity. The results for the $F 435 W, F 775 W$, and NIC2 filters are shown in Fig. 6.

In all filters the radial profiles look very similar. The polarization $Q_{\mathrm{r}}$ in the disk center at $R=0$ is essentially zero. The normalized radial polarization $Q_{\mathrm{r}} / I$ increases steadily with radius until it peaks at around $R_{\text {Titan }}=0.44^{\prime \prime}$ after which it decreases again until the photon noise starts dominating the measurements. Similarly, the radial polarization flux $Q_{\mathrm{r}}$ also increases with radius but only up to a radius slightly smaller than $R_{\text {Titan }}$, and then it decreases farther out to zero in step with the intensity profile.

Both $Q_{\mathrm{r}} / I$ and $Q_{\mathrm{r}}$ increase with wavelength until they peak in the NICl band at $1 \mu \mathrm{m}$. Then, in the NIC 2 band at $2 \mu \mathrm{m}$ the polarization has again significantly dropped. Using tentative fits for the radial profiles, we measure maximum fractional radial polarization values $Q_{\mathrm{r}} / I$ of $1.8 \%$ for the $F 250 \mathrm{~W}$ band, up to $6.9 \%$ for 
the NIC1 band, and 3.4\% for the NIC2 band. The values for all filters are given in Table 3 . However we note that these values are not yet corrected for the PSF smearing effect described in Sect. 6.4.

\subsection{Disk-integrated radial polarization}

Similar to the calculation of disk-integrated Stokes parameters in Sect. 5 disk-integrated radial Stokes parameters $\left\langle Q_{\mathrm{r}} / I\right\rangle_{\mathrm{m}}$ and $\left\langle U_{\mathrm{r}} / I\right\rangle_{\mathrm{m}}$ are calculated for all filters. Because of the intrinsic rotational symmetry of Eqs. (2)-(4), $\left\langle Q_{\mathrm{r}} / I\right\rangle_{\mathrm{m}}$ and $\left\langle U_{\mathrm{r}} / I\right\rangle_{\mathrm{m}}$ have the additional advantage that any instrumental polarization offset or gradient cancels out. Therefore, we do not need to correct the instrumental offset described in Sect. 5 for the $\mathrm{F330W}$ and $\mathrm{NICl}$ filter to calculate corresponding disk-integrated radial polarization values. This was also verified by calculating $\left\langle Q_{\mathrm{r}} / I\right\rangle_{\mathrm{m}}$ and $\left\langle U_{\mathrm{r}} / I\right\rangle_{\mathrm{m}}$ for the NICl data, both with and without correction of the instrumental offset, which showed that the difference is less than $\Delta p=0.01 \%$.

A strong positive signal is obtained for the disk-integrated radial polarization $\left\langle Q_{\mathrm{r}} / I\right\rangle_{\mathrm{m}}$, while $\left\langle U_{\mathrm{r}} / I\right\rangle_{\mathrm{m}}$ is essentially zero (Table 3). The $1 \sigma$ measuring error is $\Delta p<0.1 \%$ as estimated from the assumption $\langle U / I\rangle_{\mathrm{m}}=0$. Both $\left\langle Q_{\mathrm{r}} / I\right\rangle_{\mathrm{m}}$ and $\left\langle U_{\mathrm{r}} / I\right\rangle_{\mathrm{m}}$ are only very weakly affected by small asymmetries caused by inaccuracies in the image centering procedure or other spurious effects due to the data reduction. However, strong asymmetric perturbations such as strong ghosts could probably bias the result. Anyway, except for NICl we do not see any ghosts in our $Q_{\mathrm{r}}$ and $U_{\mathrm{r}}$ images (Sect. 6.1). In case for NICl it is known that weak ghosting is present for bright sources but we estimate that the impact on the integrated radial polarization is less than $\Delta p / p=0.01$ (see also Sect. 7.3).

The integrated radial polarization $\left\langle Q_{\mathrm{r}} / I\right\rangle$ is a good parameter for characterizing the overall limb polarization of a planet. Since $Q_{\mathrm{r}}$ is either positive or close to zero everywhere on the disk no polarization compensation effect is present. Furthermore, there is $\left\langle Q_{\mathrm{r}} / I\right\rangle \gg\left\langle U_{\mathrm{r}} / I\right\rangle \approx 0$, so that we can approximate

$$
\left\langle p_{\mathrm{r}}\right\rangle=\sqrt{\left\langle Q_{\mathrm{r}} / I\right\rangle^{2}+\left\langle U_{\mathrm{r}} / I\right\rangle^{2}} \approx\left\langle Q_{\mathrm{r}} / I\right\rangle
$$

\subsection{Correction for the PSF smearing effect}

The point spread functions (PSFs) of HST ACS and NICMOS have a finite width given by the telescope diffraction $\sim \lambda / D$, and they are affected by optical aberrations, geometric distortions, and in case of ACS a long-wavelength halo produced by the detector itself. This leads to extended PSF wings which limit the resolution of the HST observations. Therefore, the measured integrated limb polarizations $\left\langle Q_{\mathrm{r}} / I\right\rangle_{\mathrm{m}}$ and $\left\langle U_{\mathrm{r}} / I\right\rangle_{\mathrm{m}}$ need to be corrected by the inverse of a degradation factor $C_{\mathrm{PSF}}$, accounting for a polarization cancelation due to PSF smearing.

We produced simulated ACS and NICMOS PSFs for all wavelengths, using the Tiny Tim $^{4}$ PSF simulation software package for the HST (see Krist et al. 2011). Figure 7 compares the encircled energy of these PSFs to the encircled energy of Gaussian PSFs with $F W H M=\lambda / D$. One can see that for the Gaussian PSFs essentially all the energy is contained within half the radius of Titan. However, in the NIC 2 band up to $45 \%$ of the energy is smeared over an area larger than half of the radius of Titan. For the ACS filters and NICl the fraction originating from $R>0.5 R_{\text {Titan }}$ is considerably smaller but still about $15 \%$ and $20 \%$ respectively.

\footnotetext{
4 http://tinytim.stsci.edu
}

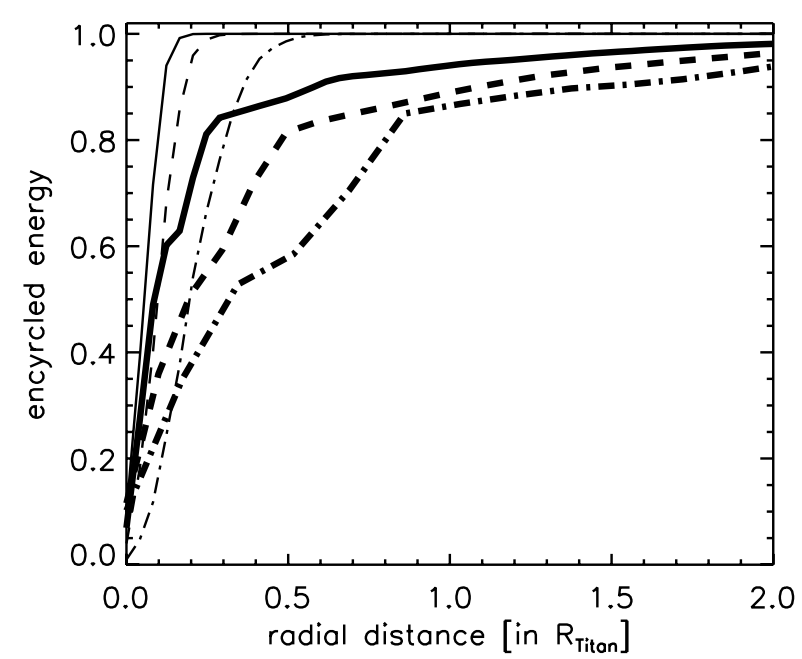

Fig. 7. Encircled energies for HST PSFs (thick lines) and Gaussian PSFs (thin lines): ACS F625W (solid), NIC1 POLOS (dashed), and NIC2 POLOL (dash-dot).

Because of these extended PSF wings, the opposite polarization components $+Q$ and $-Q$ overlap and cause a reduction in the resulting net polarization. In the most extreme case of an unresolved centro-symmetric planetary disk, the polarization cancelation would be perfect and only a zero net polarization level could be measured. The compensation effect is stronger for longer wavelengths, where the diffraction limited spatial resolution of HST is not as good, and at the same time the PSF wings are stronger.

For an estimate of the polarization cancelation, we adopted our haze scattering model for the expected polarization pattern (see Sect. 7.2). From the model we constructed two-dimensional intensity images for $i_{0}, i_{90}, i_{45}$, and $i_{135}$, from which the corresponding Stokes $Q$ and $U$ images can be calculated by $Q=$ $i_{0}-i_{90}$ and $U=i_{45}-i_{135}$. Similarly, we constructed smeared Stokes images $Q_{\mathrm{s}}$ and $U_{\mathrm{s}}$ by folding the $i_{*}$ images with the simulated HST PSFs. Figure 8 illustrates the cancelation effect in the Stokes $Q$ image due to the HST PSF of ACS at F625W, NIC1 at $1 \mu \mathrm{m}$, and NIC2 at $2 \mu \mathrm{m}$.

The $Q$ and $U$ images can then be converted into radial polarization images $Q_{\mathrm{r}}$ and $U_{\mathrm{r}}$, as for the observations. From $Q_{\mathrm{r}}$ the integrated radial polarizations $\left\langle Q_{\mathrm{r}} / I\right\rangle$ and $\left\langle Q_{\mathrm{r}} / I\right\rangle_{\mathrm{s}}$ are calculated for the different filters in the same way as for the observations. The ratio between the clean and the smeared polarization then yields the factor for the expected degradation of the diskintegrated radial polarization

$C_{\mathrm{PSF}}=\frac{\left\langle Q_{\mathrm{r}} / I\right\rangle_{\mathrm{s}}}{\left\langle Q_{\mathrm{r}} / I\right\rangle}$

The corresponding values of $C_{\mathrm{PSF}}$ and the corrected limb polarization $\left\langle Q_{\mathrm{r}} / I\right\rangle$ for all filters are given in Table 3. Especially for NIC2 the degradation factor $C_{\mathrm{PSF}}=0.58$ is low, while for the ACS bands and for NICl the degradation factor is about $C_{\mathrm{PSF}}=$ 0.85 and $C_{\mathrm{PSF}}=0.74$ respectively. The statistical $1 \sigma$ error of the degradation factor is estimated to be about $\Delta C_{\mathrm{PSF}}= \pm 0.01$.

The polarization cancelation depends not on the strength, but on the geometric structure of the polarization pattern. Rayleigh scattering models indicate that the polarization pattern is very similar for the different model parameters (see Schmid et al. 2006b). Thus the degradation depends not significantly on the exact haze scattering parameters of the planet. A sanity check by recalculating $C_{\mathrm{PSF}}$ using a Rayleigh scattering model indeed 

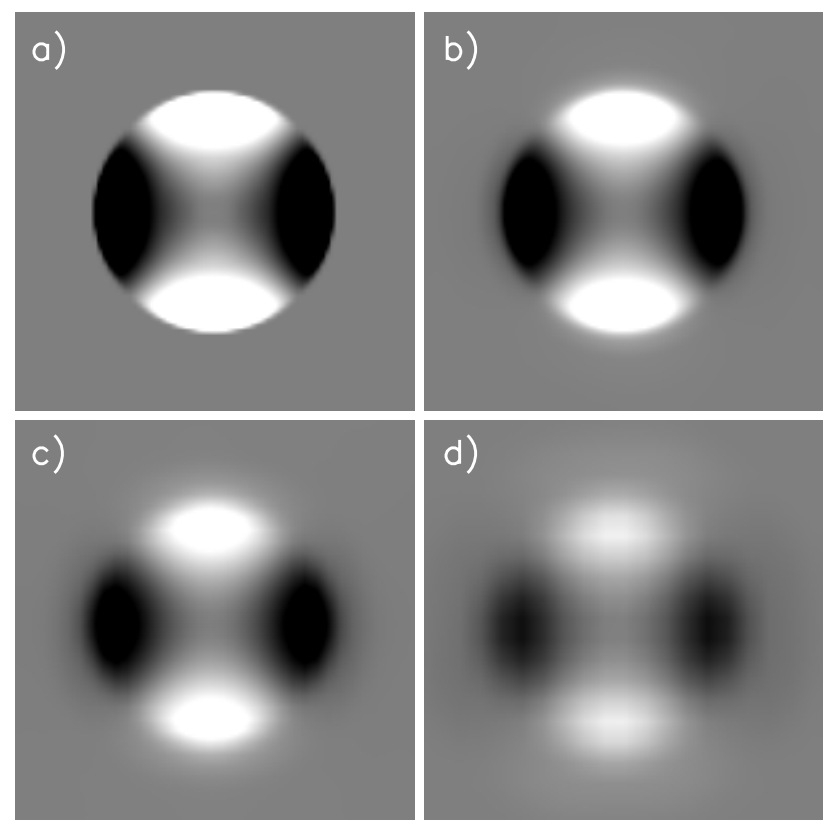

Fig. 8. Modeling of the degradation of the Titan polarization $Q$ due to the cancelation of opposite polarization components $+Q$ and $-Q$ caused by the limited resolution; a) unlimited resolution; b) HST PSF at $630 \mathrm{~nm}(F 625 W)$; c) HST PSF at $1 \mu \mathrm{m}(N I C 1)$; and d) HST PSF at $2 \mu \mathrm{m}$ (NIC2). The gray scale spans for all panels the range from $-2 \%$ (black) to $+2 \%$ (white) of the peak intensity of the initial perfect intensity image.

shows that within our $1 \sigma$ error bars the results for $C_{\mathrm{PSF}}$ were identical.

\section{Comparison with limb polarization models}

Titan is an excellent test case for detailed studies of the scattering polarization from a hazy atmosphere, and accurate scattering and polarization parameters are available from the in situ measurements of the Huygens landing probe (e.g., Brown et al. 2010; Tomasko et al. 2008). In the next section we describe the basic atmospheric structure that we used for our radiative transfer model, which is described in Sect. 7.2, and in Sect. 7.3 we compare the model with our limb polarization measurements and literature values for the geometric albedo $A_{\mathrm{g}}$ and the quadrature polarization $p\left(90^{\circ}\right)$ of Titan.

\subsection{Atmospheric parameters}

For our model we assume an atmosphere ranging from $0-1300 \mathrm{~km}$. This includes Titan's troposphere with its well defined tropopause at $\sim 44 \mathrm{~km}(112 \mathrm{mbar})$, the stratosphere with the stratopause located at $\sim 260-310 \mathrm{~km}(0.22-0.08 \mathrm{mbar}$; Fulchignoni et al. 2005; Vinatier et al. 2007), the mesosphere with the mesopause at $\sim 494 \mathrm{~km}(0.002 \mathrm{mbar}$; Fulchignoni et al. $2005)$, and the thermosphere ranging up to $\sim 1300 \mathrm{~km}(1.4 \times$ $10^{-8}$ mbar).

The atmospheric composition is predominantly $\mathrm{N}_{2}$, with $\mathrm{CH}_{4}$ and $\mathrm{H}_{2}$ the second and third most abundant molecules respectively. Near the surface $\mathrm{CH}_{4}$ has an abundance of $\sim 5 \%$, falling to $\sim 1.4 \%$ in the stratosphere (Brown et al. 2010). Because of its long chemical lifetime $\mathrm{H}_{2}$ is essentially uniformly mixed throughout the atmosphere with a mixing ratio $\sim 0.1 \%$ (Courtin et al. 2008). A thick haze layer is located in the stratosphere, and a second detached haze layer lies just above the mesopause at

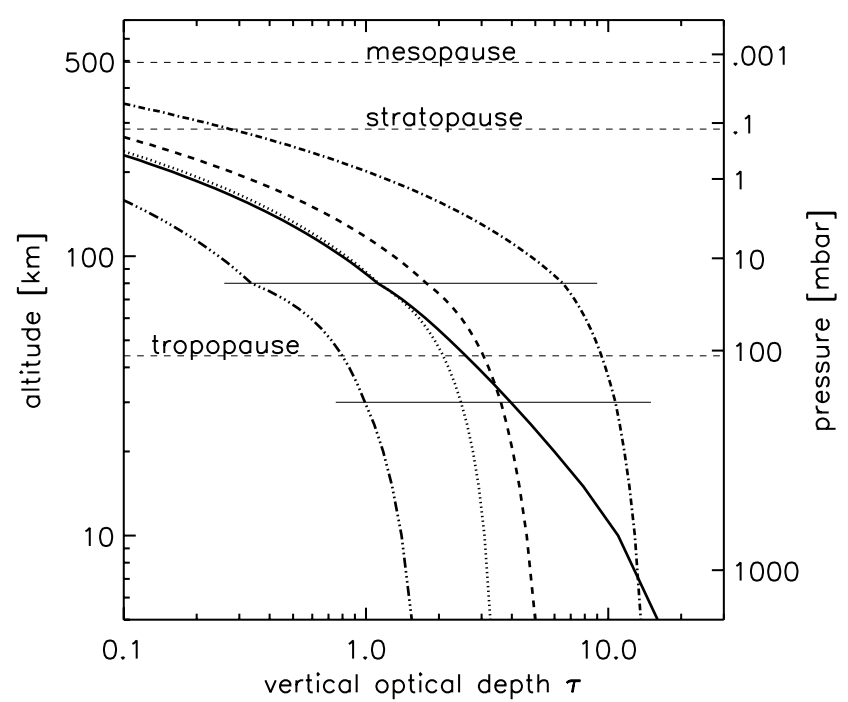

Fig. 9. Vertical optical depth $\tau$ for $\lambda=445 \mathrm{~nm}$ (dash-dot), $\lambda=775 \mathrm{~nm}$ (dash), $\lambda=940 \mathrm{~nm}$ (dot), $\lambda=1000 \mathrm{~nm}$ (methane absorption band, solid), and for $\lambda=1580 \mathrm{~nm}$.

around $500 \mathrm{~km}$ altitude (Porco et al. 2005). Above 700-800 km most of the methane is destroyed by photolysis.

For simplification, we assume the atmospheric composition to be solely made out of methane and nitrogen. The methane mole fraction $f_{\mathrm{CH}_{4}}(h)$ between $h=0-144 \mathrm{~km}$ altitude was taken from Brown et al. (2010), whereas above $140 \mathrm{~km} f_{\mathrm{CH}_{4}}(h)$ was assumed to linearly drop to zero at an altitude of $600 \mathrm{~km}$. We use the temperature $T(h)$ and density $\rho(h)$ profiles provided by the Huygens Atmospheric Structure Instrument (HASI) ${ }^{5}$, and the total molecular number densities and the Rayleigh scattering optical depth $\tau_{\text {Ray }}(h, \lambda)$ are then calculated from the ideal gas law and the gas column density (see Appendix).

For the methane absorption coefficients $\kappa(T, \lambda)$ we use the formula given by Karkoschka \& Tomasko (2010). We note that below $\lambda=1 \mu \mathrm{m}$ these coefficients are generally close to those by Karkoschka (1998).

A detailed model of the aerosol properties of Titan is given by Tomasko et al. (2008), based on measurements of the DISR instrument on the Huygens landing probe (Tomasko et al. 2002). The haze optical depth per unit path length $\tau_{\text {haze }}(h, \lambda)$ is derived for three altitude regions, i.e., above $80 \mathrm{~km}$, between $30-80 \mathrm{~km}$, and below $30 \mathrm{~km}$. On the one hand, Tomasko et al. (2008) describe the wavelength dependence by three different power laws, corresponding to the three altitude regions. Then on the other hand, the cumulative optical depth increases with decreasing altitude. Between $0-30 \mathrm{~km}$ and $30-80 \mathrm{~km}$ the increase is linear but with two different slopes, and above $80 \mathrm{~km}$ the increase is exponential with a scale height of $65 \mathrm{~km}$.

The vertical optical depth is shown in Fig. 9 for five different wavelengths between $\lambda=445 \mathrm{~nm}$ and $\lambda=1580 \mathrm{~nm}$, including the methane absorption band at $\lambda=1 \mu \mathrm{m}$. For wavelengths $\lambda<$ $1 \mu \mathrm{m}$ most of the light is scattered in the stratosphere between $\sim 100-300 \mathrm{~km}$ altitude where $\tau \approx 1$, whereas for $\lambda=1.6 \mu \mathrm{m}$ the light penetrates down to about $30 \mathrm{~km}$ altitude. The impact of the methane absorption is particularly strong in the troposphere but has almost no effect for higher altitudes.

Similar to $\tau$, the single scattering albedo $\omega_{\text {haze }}(h, \lambda)$ can be split into wavelength and altitude dependent parts, whereas

5 http://atmos.nmsu.edu/PDS/data/hphasi_0001/DATA/ PROFILES/ 
typically $\omega_{\text {haze }}(h, \lambda) \approx 0.8-1$. We adopt the altitude model of Tomasko et al. (2008). Between $0-30 \mathrm{~km}, 30-80 \mathrm{~km}$, and above $144 \mathrm{~km}$ respectively, we assume $\omega_{\text {haze }}(h, \lambda)$ to be constant with altitude, using the values given in Table 2 of Tomasko et al. (2008), whereas between $80-144 \mathrm{~km}$ we linearly interpolate between the adjacent regions. For the region between $80 \mathrm{~km}$ to $144 \mathrm{~km}$ Tomasko et al. (2008) suggest that new material is incorporated in the aerosols as they fall, and that the aerosols grow in size. In the few kilometers above the surface they also see some weak evidence of a decrease of $\omega_{\text {haze }}(h, \lambda)$, i.e., reversing the general trend with altitude. The wavelength dependence of $\omega_{\text {haze }}$ for the region above $144 \mathrm{~km}$ and $30-80 \mathrm{~km}$ is given in Fig. 48 of Tomasko et al. (2008), and the same dependence is assumed for the region 80-144 km. For the region below $30 \mathrm{~km}$ we adopt a three-dimensional polynomial fit to the values given in Table 2 of Tomasko et al. (2008).

For the surface we assume a diffusely scattering surface with constant albedo $A_{\mathrm{s}}=0.2$ for all wavelengths. This is a strong simplification and according to the literature the surface albedo of Titan varies between $A_{\mathrm{s}}=0.1-0.3$, depending on wavelength and also the season of Titan (e.g., McKay et al. 1989; Tomasko et al. 1997, 2008). Anyway, we tested different models using a range of $A_{\mathrm{s}}=0.1-0.3$, and the variation from $\left.p\left(0^{\circ}\right)\right|_{A_{\mathrm{s}}=0.2}$ is less than $\Delta p\left(0^{\circ}\right) / p\left(0^{\circ}\right) \approx 0.1$.

The wavelength range of our model is restricted by the Tomasko et al. (2008) values for $\tau_{\text {haze }}(h, \lambda)$ and $\omega_{\text {haze }}(h, \lambda)$, which are only given for a wavelength range of 400-1600 nm. On the one hand, we extended this range towards the UV by extrapolating the parameters down to $200 \mathrm{~nm}$. To first order this is valid because between $200-400 \mathrm{~nm}$ we do not expect strong spectral or altitudinal features in $\tau_{\text {haze }}(h, \lambda)$ and $\omega_{\text {haze }}(h, \lambda)$. On the other hand, we did not extrapolate the parameter range towards the red end because above $\lambda \approx 1 \mu \mathrm{m}$ the spectral variation in $\omega_{\text {haze }}$ is more complex, and because the extrapolation interval to include the NIC2 band at $2.2 \mu \mathrm{m}$ was too large. Therefore, the final spectral range of the model covers 200-1600 nm, which includes all our polarization data except the NIC 2 polarimetry.

\subsection{Radiative transfer code}

We use an extended version of the Monte Carlo scattering code described by Buenzli \& Schmid (2009). Basically, the code calculates random walk histories of many photons entering the atmosphere, and follows their direction and polarization change until they are absorbed or they escape. The intensity and polarization spectra of the planet can then be established for different lines of sight, and in the case for backscattering $\left(\alpha=0^{\circ}\right)$ as a function of radial distance from the disk center. For the calculation, the spherical model atmosphere is assumed to be rotationally homogeneous, consisting of multiple locally plane parallel layers. The incident radiation is a parallel beam of unpolarized photons, whereas despite multiple scattering, the photons emerge at the same point where they entered into the atmosphere.

The scattering processes are described by probability density functions, derived from the appropriate phase matrices of the scattering particles (see also Schmid 1992). For scattering on haze particles, the code allows for scattering matrices of the form

$\mathbf{F}(\theta)=\left(\begin{array}{cccc}F_{11}(\theta) & F_{12}(\theta) & 0 & 0 \\ F_{12}(\theta) & F_{11}(\theta) & 0 & 0 \\ 0 & 0 & F_{33}(\theta) & 0 \\ 0 & 0 & 0 & 0\end{array}\right)$,

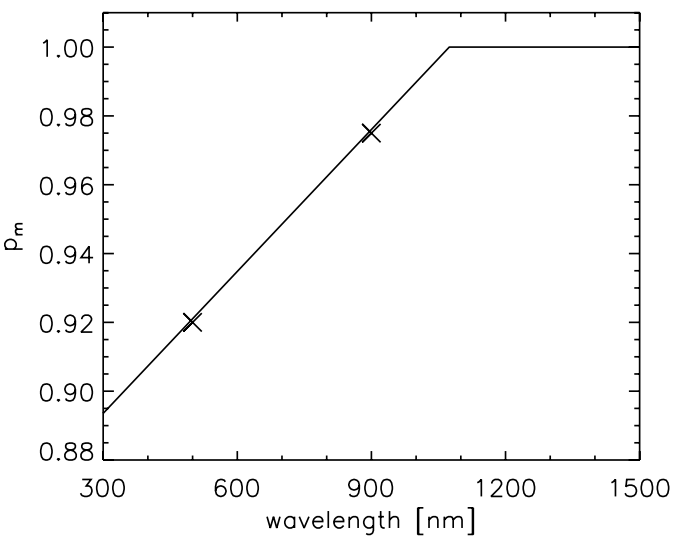

Fig. 10. Wavelength dependence of the single scattering polarization parameter $p_{m}$ defined by Eq. (7). The fit values to the Tomasko et al. (2008) curves for the blue and the red channel are also indicated.

where $\theta$ is the scattering angle. The code does not consider circular polarization because these effects are expected to be very small and negligible for a simple scattering model.

We used the aerosol scattering phase functions $F_{11}(\theta)$ given in tabulated form by Tomasko et al. (2008) for two altitude regions, above $80 \mathrm{~km}$ and below $80 \mathrm{~km}$, and for different wavelengths ranging between $355 \mathrm{~nm}$ to $5166 \mathrm{~nm}$. Typically a phase function is given about every $100 \mathrm{~nm}$ for $\lambda<1 \mu \mathrm{m}$ and about every $200 \mathrm{~nm}$ for $1 \mu \mathrm{m}<\lambda<1.5 \mu \mathrm{m}$, and for our model calculations we linearly interpolate between these values. Anyway, it turned out that for the polarization results the dependence on $\lambda$ is very weak. This was tested by running different model implementations using only the tabulated phase functions with the closest match to $\lambda$, and within our observational error bars the calculated limb polarization was the same.

Tomasko et al. (2008) also derive a single scattering polarization fraction $\left(-F_{12} / F_{11}\right)$ for the blue $(470-530 \mathrm{~nm})$ and the red $(880-970 \mathrm{~nm})$. To first order, we find a very tight fit to their model using a scaled Rayleigh-like single scattering polarization dependence according to

$\frac{F_{12}(\theta)}{F_{11}(\theta)}=p_{m} \frac{\cos ^{2}(\theta)-1}{\cos ^{2}(\theta)+1}$

with $p_{m}=0.920$ for the blue channel and $p_{m}=0.975$ for the red channel. For our model we use $p_{m}(\lambda)$ with a linear slope, going through the values of the red and blue channel, and $p_{m}(\lambda)=1$ above $\lambda \approx 1 \mu \mathrm{m}$ as shown in Fig. 10 .

For $F_{33}(\theta)$ we use the same dependence as for Rayleigh scattering according to

$\frac{F_{33}(\theta)}{F_{11}(\theta)}=\frac{2 \cos (\theta)}{\cos ^{2}(\theta)+1}$.

The atmospheric parameters used for our model are described in the previous section. Our calculations include Rayleigh scattering, aerosol scattering, and methane absorption but we neglect Raman scattering, which has only a very small effect on the reflectivity and polarization (e.g., Sromovsky 2005). The model atmosphere consists of 47 different layers above a diffusely scattering surface, and the models are run with $10^{9}-10^{10}$ photons depending on wavelength, such that the statistical error of the fractional polarization is $\Delta p \leq \pm 0.1 \%$. 


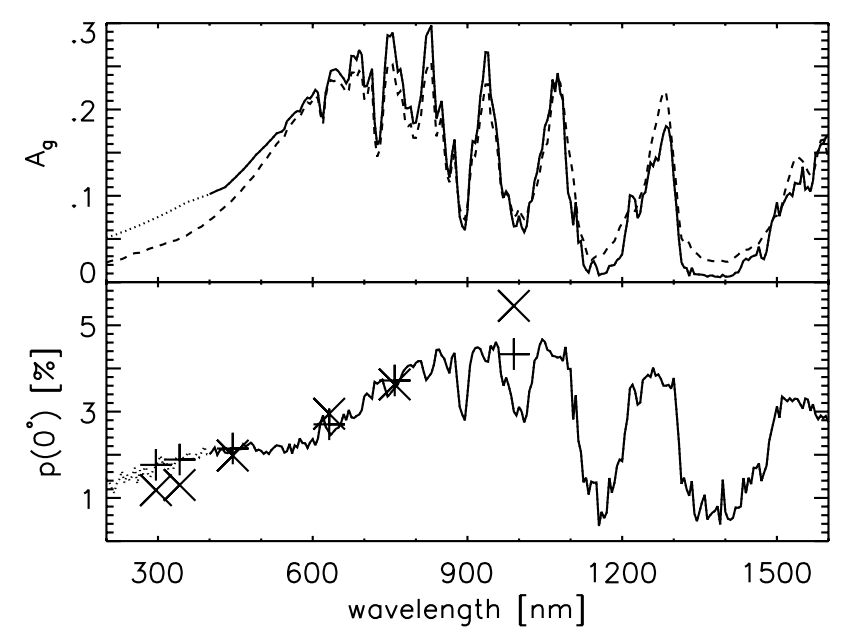

Fig. 11. Comparison of our model results for the limb polarization with literature values. Top panel: geometric albedo $A_{\mathrm{g}}$ from the literature (dashed, see also Fig. 1) and from our model (solid). Bottom panel: integrated limb polarization $p\left(0^{\circ}\right)$ of our model for the complete wavelength range (solid), and the HST filter pass bands (+), as well as our HST polarimetry observations $(\times)$ given in Table 3.

\subsection{Results}

Our model calculates the geometric albedo $A_{\mathrm{g}}$, the quadrature polarization $p\left(90^{\circ}\right)=\langle Q / I\rangle\left(90^{\circ}\right)$, and the limb polarization $p\left(0^{\circ}\right)=\left\langle Q_{\mathrm{r}} / I\right\rangle$. Figure 11 compares in the top panel the calculated geometric albedo with observational data (see Sect. 2). We find a good qualitative agreement for the complete wavelength range. Above $500 \mathrm{~nm}$ the agreement outside strong absorption bands is better than $\Delta A_{\mathrm{g}} / A_{\mathrm{g}}=0.2$, whereas for the strong absorption around $1.15 \mu \mathrm{m}$ and $1.45 \mu \mathrm{m}$ the agreement is $\Delta A_{\mathrm{g}} / A_{\mathrm{g}} \approx 0.3$. Below $500 \mathrm{~nm}$ the agreement gets worse with decreasing wavelength. At $400 \mathrm{~nm}$ it is $\Delta A_{\mathrm{g}} / A_{\mathrm{g}} \approx 0.4$ and at $300 \mathrm{~nm}$ it is only $\Delta A_{\mathrm{g}} / A_{\mathrm{g}} \approx 0.75$. We note that the disagreement below $400 \mathrm{~nm}$ is not alarming because there the albedo is very low. Furthermore, it could also origin from our questionable extrapolation of the Tomasko et al. (2008) haze parameters from $400 \mathrm{~nm}$ to $200 \mathrm{~nm}$.

The limb polarization results for the model and our measurements are shown in the bottom panel of Fig. 11. Between $400-900 \mathrm{~nm}$ the agreement is good with $\Delta p / p \approx 0.1$ for the $F 625 W$ pass band, and $\Delta p / p \leq 0.05$ for the $F 435 W$ and $F 775$ pass bands. Below $400 \mathrm{~nm}$ the model seems to systematically overestimate the polarization, yielding a discrepancy of $\Delta p / p \approx 0.3$. Qualitatively this UV-offset agrees with our result for the geometric albedo $A_{\mathrm{g}}$, which also seems to be systematically too high in the UV. Above $900 \mathrm{~nm}$ our NICl polarization is much higher than the model result and $\Delta p / p \approx 0.25$. We could not conclusively determine whether this discrepancy is caused by an issue of the measurement, the modeling, or both.

It is known that the polarimetric calibration of $\mathrm{NICl}$ has some deficiencies such as residual instrumental polarization of $p_{\text {inst. }} \sim 1.5 \%$ and weak ghosting (see Sect. 3.2). However, because of the intrinsic symmetry of the radial Stokes parameters $Q_{\mathrm{r}}$ and $U_{\mathrm{r}}$ the residual instrument polarization has no effect on $\left\langle Q_{\mathrm{r}} / I\right\rangle$ (see Sect. 6.3). The ghosting on the other hand is asymmetric and thus could have an impact on $\left\langle Q_{\mathrm{r}} / I\right\rangle$. However, in our data we only see very weak ghosts which we do not believe to have an effect at the percent level. Finally, it could also be that the real PSF is different from the adopted PSF used for the efficiency correction $C_{\mathrm{PSF}}$ (Sect. 6.4). An overcompensation

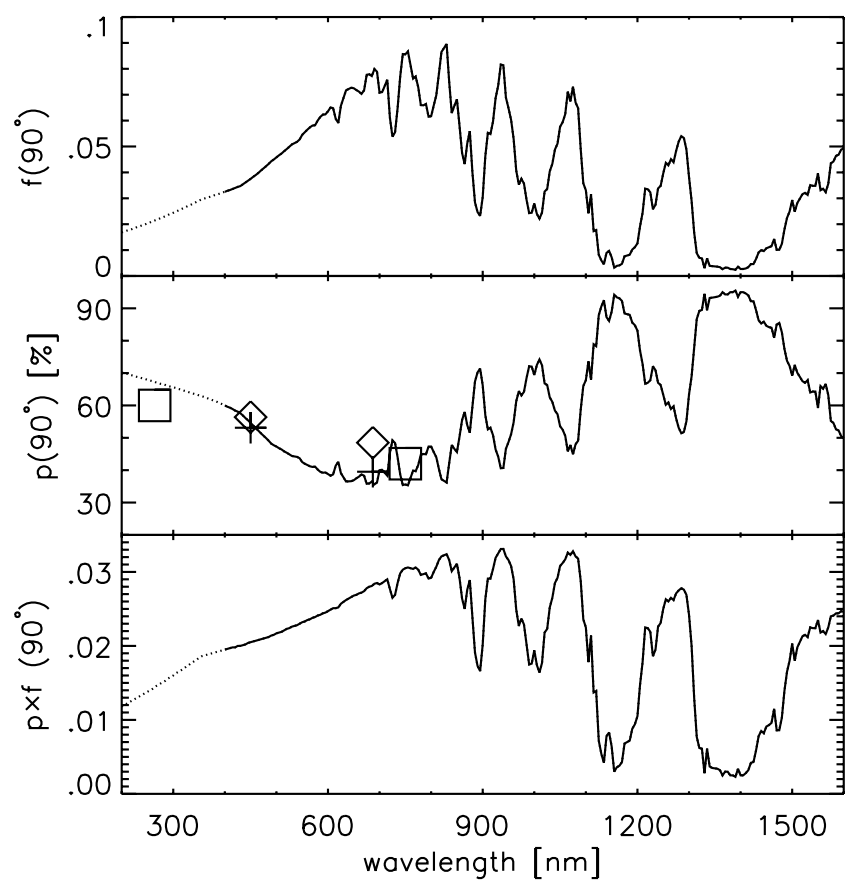

Fig. 12. Comparison of our model results at quadrature phase $\alpha=90^{\circ}$ with literature values. Top panel: reflectivity $f\left(90^{\circ}\right)$. Middle panel: integrated quadrature polarization $p\left(90^{\circ}\right)$ of our model for the complete wavelength range (solid), and the results from the Pioneer $11(\diamond)$ and Voyager $2(\square)$ spacecrafts. The model results in the Pioneer 11 pass bands are also indicated (+). Bottom panel: integrated polarization flux $p \times f\left(90^{\circ}\right)$.

of the PSF smearing could explain a $\Delta p / p$ of a few percent but certainly not the full discrepancy between model and data.

Concerning the model parameters for the $\mathrm{NICl}$ wavelength range and their impact on $\left\langle Q_{\mathrm{r}} / I\right\rangle$, we tested the effect of the surface albedo $A_{\mathrm{s}}$, the single scattering polarization parameter $p_{m}$, and the methane fraction as functions of altitude. Using a range of $A_{\mathrm{S}}=0.1-0.3$ showed that the impact of $A_{\mathrm{S}}$ is less than $\Delta p / p \approx 0.1$. Similarly, setting $p_{m}=1$ everywhere cannot explain the discrepancy and the effect is less than $\Delta p / p \approx 0.05$. Finally, we set the methane mole fraction $f_{\mathrm{CH}_{4}}=0$ for $h>80 \mathrm{~km}$ to check the impact of the methane absorption in the stratosphere and mesosphere. This basically gets rid of the absorption dips in $\left\langle Q_{\mathrm{r}} / I\right\rangle$ but only has a minor impact outside the absorption bands. Since $\left\langle Q_{\mathrm{r}} / I\right\rangle$ is flux weighted, we conclude that the overall impact of the methane absorption on the limb polarization is much less than $\Delta p / p \approx 0.1$. Therefore, the cause of the disagreement between the model and the NICl measurement remains uncertain.

Titan full disk phase curves for intensity and polarization have been obtained by the Pioneer 11 (Tomasko \& Smith 1982) and the Voyager 2 (West et al. 1983) spacecrafts. The Pioneer 11 data were obtained in the $B$ and $R$ bands, covering phase angles between $\alpha=28^{\circ}-96^{\circ}$, whereas the Voyager 2 data were taken in the near UV at $264 \mathrm{~nm}$ and the near IR at $750 \mathrm{~nm}$, covering phase angles $\alpha=2.7^{\circ}-154^{\circ}$. The second panel of Fig. 12 compares our model with the quadrature polarization at $\alpha=90^{\circ}$ measured by Pioneer 11 and Voyager 2. For three bands we find a good agreement better than $\Delta p / p \approx 0.1$, whereas for the Pioneer $11 R$ band the agreement is not as tight but still at the level $\Delta p / p \approx 0.25$.

We note that the quadrature polarization $p\left(90^{\circ}\right)$ is generally higher in the absorption bands because multiple scattering is 
strongly reduced, and the reflection is dominated by strongly polarized single scattering at $\sim 90^{\circ}$. For the limb polarization $p\left(0^{\circ}\right)$ this $A_{\mathrm{g}}-p$ correlation is reversed. Multiple scattering and a low single scattering albedo $\omega$ are required for producing a strong polarization signal since the total polarization is mainly produced by second order but also higher order scatterings.

\section{Discussion and conclusions}

We present disk resolved imaging polarimetry of Titan, measured with the HST for the UV to the near-IR spectral region. From these observations, we derive the disk-integrated radial limb polarization $\left\langle Q_{\mathrm{r}} / I\right\rangle$ (Table 3 ) for various filter pass bands, and we compare our results with the polarization of a model atmosphere. For the model we use a radiative transfer code presented in Buenzli \& Schmid (2009), adopting Titan atmosphere parameters from the literature, which were mainly derived from the Huygens landing probe (e.g., Tomasko et al. 2008).

The geometric albedo $A_{\mathrm{g}}$ and the quadrature polarization $p\left(90^{\circ}\right)$ are important reference quantities for characterizing the scattering properties of a reflecting atmosphere. Therefore, we derive the reflected-flux weighted geometric albedo $A_{\mathrm{g} \text {,eff }}$ for the used filter pass bands (Table 3), using the spectrophotometry of McGrath et al. (1998), Karkoschka (1998), and Negrão et al. (2006); and we compare our model results for $p\left(90^{\circ}\right)$ with measurements obtained by the Pioneer 11 (Tomasko \& Smith 1982) and the Voyager 2 (West et al. 1983) spacecrafts.

A comparison between the model and our observations for $\left\langle Q_{\mathrm{r}} / I\right\rangle(\lambda)$, as well as a comparison between our model and literature values for the geometric albedo $A_{\mathrm{g}}(\lambda)$ and the quadrature polarization $p\left(90^{\circ}, \lambda\right)$ are given in Figs. 11 and 12 .

\subsection{Detection of the limb polarization.}

For all observed filter bands, our data show a strong limb polarization of several percent, as expected from previous polarization measurements of Titan taken at larger phase angles. To our knowledge, this is the first time that the limb polarization of Titan has been measured, and we did not find any previous earth-bound imaging polarimetry which resolved Titan.

Within the resolution limits of the observations, the measured limb polarization for Titan is centro-symmetric. This is similar to observations of Uranus and Neptune (Schmid et al. 2006b) but for Titan the polarization is much stronger. On the other hand, similar polarization strength can be found in observations of Jupiter but there the limb polarization is essentially only present at the poles (e.g., Schmid et al. 2011), indicating thick polar haze layers and non-polarizing reflection from the clouds along the equator.

The polarimetric sensitivity and the resolution of our data are not sufficient to detect variations of the polarization signal along the limb of Titan, as it is seen in albedo observations. In Sect. 6.2 we pointed to some tentative limb polarization structure, which could be present in our data. Additional observations of Titan with increased polarimetric sensitivity and resolution are required to progress in this direction.

Assuming rotational symmetry, we derive center-to-limb profiles for the radial Stokes parameter $Q_{\mathrm{r}}(r) / I(r)$. Because of the scattering symmetry, $Q_{\mathrm{r}}(r) / I(r)$ is essentially zero in the disk center, whereas the polarization increases for larger radii, reaching a maximum in the seeing halo at $R_{\max }>R_{\text {Titan. }}$. Depending on wavelength, we measured maximum fractional polarization values in the range of $\sim 2-7 \%$ with the highest value obtained in the NIC1 band at $1 \mu \mathrm{m}$.

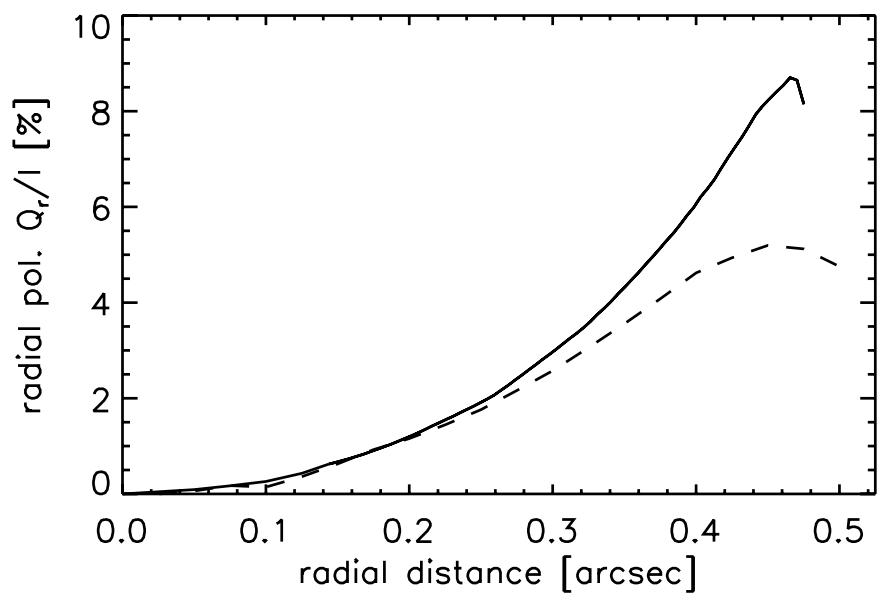

Fig. 13. Measured radial polarization profile for Titan in the $F 775 \mathrm{~W}$ band (dashed) versus the modeled profile without PSF smearing (solid).

The observed maximum limb polarization is averaged down by the limited resolution and the PSF structure of HST. This has been taken into account in our analysis of the measured diskintegrated radial polarization $\left\langle Q_{\mathrm{r}} / I\right\rangle_{\mathrm{m}}$. Using synthetic PSF profiles for HST we have modeled the resolution effect on the polarization, and we derive corrected values for the intrinsic $\left\langle Q_{\mathrm{r}} / I\right\rangle$ of Titan (Table 3). We find $\left\langle Q_{\mathrm{r}} / I\right\rangle \approx 1.2 \%$ in the UV, increasing to about $5.5 \%$ at $1 \mu \mathrm{m}$, and then decreasing again to about $3.4 \%$ at $2 \mu \mathrm{m}$. For comparison, a semi-infinite, conservative $(\omega=1)$ Rayleigh-scattering atmosphere produces a disk-integrated limb polarization $\left\langle Q_{\mathrm{r}} / I\right\rangle \approx 2.75 \%$ (Buenzli \& Schmid 2009).

Using our radiative transfer model for Titan we predict the maximum limb polarization without PSF smearing, e.g., for observations with larger earth-bound telescopes or from spacecrafts close to Titan. We find $\left(Q_{\mathrm{r}} / I\right)^{\mathrm{max}} \approx 4.2 \%$ for the $F 250 \mathrm{~W}$ band, up to $\left(Q_{\mathrm{r}} / I\right)^{\max } \approx 8.7 \%$ for the $F 775 \mathrm{~W}$ and $\left(Q_{\mathrm{r}} / I\right)^{\max } \approx$ $10.5 \%$ for the NICl band, which is even larger than $\approx 8 \%$ for a semi-infinite conservative Rayleigh-scattering atmosphere (Buenzli \& Schmid 2009). The modeled $\left(Q_{\mathrm{r}} / I\right)^{\max }$ for all filters are also given in Table 3, and Fig. 13 compares the measured $F 775 \mathrm{~W}$ radial polarization profile with the model result. In the figure one can see both the polarization dilution, as well as the decreased resolution provided by the HST.

\subsection{Comparison with model calculations}

Our model calculates the intensity and the polarization spectra of the planet at different phase angles $\alpha=0^{\circ}-180^{\circ}$, and in case of backscattering $\left(\alpha=0^{\circ}\right)$, the limb polarization as a function of the radial distance from the disk center.

Overall, we find a good agreement at the level of $\left(\Delta A_{\mathrm{g}}\right) / A_{\mathrm{g}} \approx$ $0.1-0.2$ or $(\Delta p) / p \approx 0.1$ respectively between model, literature values, and our observations. In one filter (NIC1 at $1 \mu \mathrm{m})$ we find a discrepancy of $\Delta p / p=0.25$ between the measured limb polarization $\left\langle Q_{\mathrm{r}} / I\right\rangle$ and our model, for which the cause is still uncertain. Despite this outlier, our analysis shows that limb-polarization measurements potentially offer an additional diagnostic tool for investigating the properties of scattering particles in Titan with earth-bound observations. Since our model assumes a rotationally homogeneous atmosphere, our results further show that the locally derived haze and atmosphere parameters from the Huygens probe are indeed representative for Titan. 
Additional observations of Titan with increased resolution and a high polarimetric sensitivity better than $0.1 \%$, e.g., using the upcoming SPHERE instrument at the VLT (Beuzit et al. 2008), might reveal structure along the limb and temporal changes in the polarization. Such limb polarization measurements could be useful for investigating local haze properties of Titan, and the impact of short-term and seasonal variations. In particular, the limb polarization is very sensitive to the maximum haze single scattering polarization $p_{m}$, which is strongly depending on the monomer size of the small haze aggregates (e.g., Tomasko et al. 2008). Changing $p_{m}$ in the $F 775 \mathrm{~W}$ band from, e.g., $p_{m}=0.96$ to $p_{m}=0.94$, i.e., corresponding to an increase of the monomer radius of about $15 \%$ (rough estimate based on Fig. 18 by Tomasko et al. 2008), reduces the limb polarization by $\Delta p / p \approx 0.1$, while the albedo remains essentially unchanged.

For other atmospheric parameters like $\tau_{\text {haze }}, \omega_{\text {haze }}$, or the $\mathrm{CH}_{4}$ fraction the geometric albedo $A_{\mathrm{g}}$ and the limb polarization $Q_{\mathrm{r}} / I$ will change together $\left(\omega_{\text {haze }}\right)$ or in opposite direction $\left(\tau_{\text {haze }}\right)$. Thus no structure in the limb polarization is expected if there is no strong albedo feature, like a north-south asymmetry.

\subsection{Prospects for extra-solar planets}

Differential polarimetric imaging is a particularly promising technique for the detection and characterization of extra-solar planets. With sensitive polarimetry the measurable contrast between star and planet can be enhanced by searching for the polarized signal of the scattered light from the planet within the halo of the unpolarized light from the star (e.g., Schmid et al. 2006b). The upcoming planet finder instruments SPHERE (Beuzit et al. 2008) and GPI (Macintosh et al. 2012) will both provide improved performance for substantial progress in this direction.

The measurable polarization contrast can be described by

$C_{\mathrm{p}}(\alpha, \lambda)=p(\alpha, \lambda) f(\alpha, \lambda)\left(R_{\mathrm{p}} / d_{\mathrm{p}}\right)^{2}$,

where $\alpha$ is the phase angle, $R_{\mathrm{p}}$ the radius of the planet, $d_{\mathrm{p}}$ its separation to the star, $f(\alpha, \lambda)$ is the phase-dependent reflectivity, and $p(\alpha, \lambda)$ is the integrated fractional polarization. Therefore, the investigation of $p(\alpha, \lambda)$ and the polarization flux $p(\alpha, \lambda) \times f(\alpha, \lambda)$ of Titan is important for planning future observing projects on extrasolar planetary systems and interpreting observational data.

On the one hand, Titan shows that atmospheres with thick layers of small aggregate haze particles produce a very strong polarization signal of $p\left(\alpha \approx 90^{\circ}\right) \approx 50 \%$ over a wide wavelength range from the UV at $300 \mathrm{~nm}$ to the near-IR at $2 \mu \mathrm{m}$. The bottom panel of Fig. 12 gives the expected polarized flux $p \times f$ of Titan if we would observe the object at quadrature phase $\alpha=90^{\circ}$. In the optical $p \times f\left(90^{\circ}\right)$ is increasing with wavelength with $p \times f\left(90^{\circ}\right) \approx 0.02$ at $\lambda=450 \mathrm{~nm}$, up to $p \times f\left(90^{\circ}\right) \gtrsim 0.03$ at $\lambda=850 \mathrm{~nm}$. Above $850 \mathrm{~nm}$ the polarized flux is strongly decreased in the methane absorption bands. Therefore, planets with hazy atmospheres and aerosol properties similar to Titan could be particularly good candidates for detection with ZIMPOL/SPHERE (Beuzit et al. 2008; Schmid et al. 2006a) because of their large polarization over the entire wavelength range of the instrument (520-900 nm).

On the other hand, the polarization signal could be strongly reduced for a planet with a Titan-like atmosphere but consisting of larger aggregates. If the monomers were about a factor of two larger then the single scattering polarization in the $F 775 \mathrm{~W}$ band is reduced by about $\Delta p_{m} / p_{m} \approx 0.2$ (rough estimate based on Tomasko et al. 2008), and the quadrature polarization will be reduced by $\Delta p / p \approx 0.4$ to about $p\left(90^{\circ}\right) \approx 25 \%$. The polarization signal of a pure Rayleigh scattering atmosphere would also be different with a high signal in shorter wavelength bands between 550-700 nm, and a significantly lower signal for the longer filter bands between 700-800 nm (Buenzli \& Schmid 2009). Therefore, a good knowledge of the polarization properties of the hazy atmosphere of Titan is useful for the search and investigation of the polarimetric signal of extra-solar planets.

Acknowledgements. We thank the referee for very thoughtful comments and suggestions which lead to a much improved revised version of the paper. Part of this work was supported by the FINES research fund by a grant through the Swiss National Science Foundation (SNF).

\section{Appendix A: Titan scattering model parameters}

\section{A.1. Number densities and column density}

For the calculation of the methane number density $n_{\mathrm{CH}_{4}}$ and the scale height $Z_{\mathrm{CH}_{4}}$ the following formulas are used:

$$
\begin{aligned}
n_{\mathrm{CH}_{4}} & =\frac{\rho\left[\mathrm{g} / \mathrm{cm}^{3}\right] \times 6.02 \times 10^{23}}{28 / f_{\mathrm{CH}_{4}}-12} \\
n_{\mathrm{N}_{2}} & =n_{\mathrm{CH}_{4}} \cdot \frac{f_{\mathrm{N}_{2}}}{f_{\mathrm{CH}_{4}}}=n_{\mathrm{CH}_{4}} \cdot \frac{1-f_{\mathrm{CH}_{4}}}{f_{\mathrm{CH}_{4}}} .
\end{aligned}
$$

Derivation:

$$
\begin{aligned}
& -n_{\mathrm{tot}}=\frac{\rho\left[\mathrm{g} / \mathrm{cm}^{3}\right] \cdot \mathrm{N}_{\mathrm{A}}}{\mu}=n_{\mathrm{CH}_{4}}+n_{\mathrm{N}_{2}} \\
& -\mu=28 \cdot\left(1-f_{\mathrm{CH}_{4}}\right)+16 \cdot f_{\mathrm{CH}_{4}} \\
& -n_{\mathrm{CH}_{4}}=f_{\mathrm{CH}_{4}} n_{\mathrm{tot}}=\frac{f_{\mathrm{CH}_{4}} \rho\left[\mathrm{g} / \mathrm{cm}^{3}\right] \cdot \mathrm{N}_{\mathrm{A}}}{28\left(1-f_{\mathrm{CH}_{4}}\right)+16 f_{\mathrm{CH}_{4}}} .
\end{aligned}
$$

Column density in km-am ${ }^{6}$ :

$$
Z\left[1 / \mathrm{km}^{2}\right]=10^{15} \int \mathrm{n}\left[1 / \mathrm{cm}^{3}\right] \mathrm{dh}=2.687 \times 10^{34} \times \mathrm{Z}[\mathrm{km}-\mathrm{am}] .
$$

\section{A.2. Rayleigh scattering optical depth}

This is from $\operatorname{PDS}^{7}$ :

$\tau_{\text {ray }, \text { sc }}=\tau_{1}\left(\mathrm{H}_{2}\right) \cdot\left(10.1509 \cdot \mathrm{Z}_{\mathrm{CH}_{4}}+4.6035 \cdot \mathrm{Z}_{\mathrm{N}_{2}}\right)$,

with $Z_{\mathrm{i}}[\mathrm{km}-\mathrm{am}]$ and the optical depth per km-am of hydrogen given as $(\lambda[\AA])$ :

$$
\tau_{1}\left(\mathrm{H}_{2}\right)=2.687 \cdot\left(\frac{8.14 \times 10^{11}}{\lambda^{4}}+\frac{1.28 \times 10^{18}}{\lambda^{6}}+\frac{1.61 \times 10^{24}}{\lambda^{8}}\right) .
$$

\section{A.3. Methane absorption}

Methane absorption from Karkoschka \& Tomasko (2010). Below $1 \mu \mathrm{m}$ these methane absorption coefficients are generally close to those by Karkoschka (1998),

$$
\begin{aligned}
\log \kappa(T)= & 0.5 z(z-1) \log \kappa(100)+\left(1-z^{2}\right) \log \kappa(198) \\
& +0.5 z(z+1) \log \kappa(296)
\end{aligned}
$$

\footnotetext{
$61 \mathrm{~km}-\mathrm{am}=2.687 \times 10^{24}$ molecules $\mathrm{cm}^{-2}$

7 http://pds-atmospheres.nmsu.edu/education_and_ outreach/
} 
with $z=(T-198) / 98$; and $\kappa(100), \kappa(198)$, and $\kappa(296)$ are given in Table 4 of the supplementary material of Karkoschka \& Tomasko (2010). Therefore, we get

$\tau_{\mathrm{CH}_{4}}=\kappa_{\mathrm{CH}_{4}} \cdot Z_{\mathrm{CH}_{4}}$.

\section{A.4. Haze optical depth and single scattering albedo}

The haze optical depth $\tau_{\mathrm{h}}$ and single scattering albedo $\omega_{\text {haze }}$ are taken from Tomasko et al. (2008). $\tau_{\mathrm{h}}$ is given for three altitude regions, above $80 \mathrm{~km}, 30-80 \mathrm{~km}$, and below $30 \mathrm{~km}$ (see Figs. 47 , $50)$. Above $80 \mathrm{~km}$, the cumulative optical depth increases with decreasing altitude with a scale height of $65 \mathrm{~km}$. Between 80 and $30 \mathrm{~km}$, and below $30 \mathrm{~km}$ the variation is linear with two different slopes. The wavelength dependence for the three regions is taken from Fig. 47 of Tomasko et al. (2008):

$$
\begin{aligned}
& \tau_{80}(\lambda)=1.012 \times 10^{7} \times \lambda^{-2.339} \\
& \tau_{30}(\lambda)=2.029 \times 10^{4} \times \lambda^{-1.409} \\
& \tau_{0}(\lambda)=6.270 \times 10^{2} \times \lambda^{-0.9706} \\
& \tau_{>80}(h, \lambda)=\tau_{80}(\lambda) \cdot e^{-\frac{h-80 \mathrm{~km}}{65 \mathrm{~km}}} \\
& \tau_{30-80}(h, \lambda)=\tau_{80}(\lambda)+\tau_{30}(\lambda)\left(1-\frac{h-30 \mathrm{~km}}{50 \mathrm{~km}}\right) \\
& \tau_{<30}(h, \lambda)=\tau_{80}(\lambda)+\tau_{30}(\lambda)+\tau_{0}(\lambda)\left(1-\frac{h}{30 \mathrm{~km}}\right) .
\end{aligned}
$$

The single scattering albedo $\omega_{\text {haze }}$ is given in Tomasko et al. (2008) for three altitude regions (Fig. 48, Table 2), above $144 \mathrm{~km}, 30-80 \mathrm{~km}$, and below $30 \mathrm{~km}$. The wavelength dependence of $\omega_{\text {haze }}$ for the region above $144 \mathrm{~km}$ and $30-80 \mathrm{~km}$ is given in Fig. 48 of Tomasko et al. (2008) whereas for the region below $30 \mathrm{~km}$ we adopt a three-dimensional polynomial fit to the values given in Table 2 of Tomasko et al. (2008). For the regions below $30 \mathrm{~km}, 30-80 \mathrm{~km}$, and above $144 \mathrm{~km}$, we assume $\omega_{\text {haze }}$ to be constant with altitude, whereas for the region $80-144 \mathrm{~km}$ we linearly interpolate between the values at $80 \mathrm{~km}$ and $144 \mathrm{~km}$ :

$$
\begin{aligned}
& \omega_{\text {haze },>144}(h, \lambda)=\omega_{\text {haze }, 144}(\lambda) \\
& \omega_{\text {haze }, 80-144}(h, \lambda)=\omega_{\text {haze }, 30-80}(\lambda) \\
& +\frac{\omega_{\text {haze }, 144}-\omega_{\text {haze }, 30-80}}{64 \mathrm{~km}} \cdot(h-80 \mathrm{~km}) \\
& \omega_{\text {haze }, 30-80}(h, \lambda)=\omega_{\text {haze }, 30-80}(\lambda) \\
& \omega_{\text {haze },<30}(h, \lambda)=\omega_{\text {haze },<30}(\lambda) \text {. }
\end{aligned}
$$

\section{A.5. Effective single scattering albedo}

$$
\begin{aligned}
& \tau_{\text {tot }}=\tau_{\text {ray,sc }}+\tau_{\mathrm{CH}_{4}}+\tau_{\text {haze }} \\
& \omega_{\text {ray }}=\tau_{\text {ray,sc }} /\left(\tau_{\text {ray,sc }}+\tau_{\mathrm{CH}_{4}}\right) \\
& \omega_{\text {ray,eff }}=\tau_{\text {ray,sc }} / \tau_{\text {tot }} \\
& \omega_{\text {haze,eff }}=\left(\omega_{\text {haze }} \cdot \tau_{\text {haze }}\right) / \tau_{\text {tot }} .
\end{aligned}
$$

\section{References}

Batcheldor, D., Schneider, G., Hines, D. C., et al. 2009, PASP, 121, 153 Beuzit, J.-L., Feldt, M., Dohlen, K., et al. 2008, in SPIE Conf. Ser., 7014, 18 Brown, R. H., Lebreton, J.-P., \& Waite, J. H. 2010, Titan from Cassini-Huygens (Springer Science)

Buenzli, E., \& Schmid, H. M. 2009, A\&A, 504, 259

Courtin, R. D., Sim, C., Kim, S., Gautier, D., \& Jennings, D. E. 2008, BAAS, 40,446

Fulchignoni, M., Ferri, F., Angrilli, F., et al. 2005, Nature, 438, 785

Karkoschka, E. 1998, Icarus, 133, 134

Karkoschka, E., \& Tomasko, M. G. 2010, Icarus, 205, 674

Krist, J. E., Hook, R. N., \& Stoehr, F. 2011, in SPIE Conf. Ser., 8127

Lorenz, R. D., Smith, P. H., \& Lemmon, M. T. 2004, Geophys. Res. Lett., 31, 10702

Lorenz, R. D., Lemmon, M. T., \& Smith, P. H. 2006, MNRAS, 369, 1683

Macintosh, B. A., Anthony, A., Atwood, J., et al. 2012, in SPIE Conf. Ser., 8446

McGrath, M. A., Courtin, R., Smith, T. E., Feldman, P. D., \& Strobel, D. F. 1998, Icarus, 131,382

McKay, C. P., Pollack, J. B., \& Courtin, R. 1989, Icarus, 80, 23

Negrão, A., Coustenis, A., Lellouch, E., et al. 2006, Planet. Space Sci., 54, 1225

Porco, C. C., Baker, E., Barbara, J., et al. 2005, Nature, 434, 159

Rannou, P., Hourdin, F., \& McKay, C. P. 2002, Nature, 418, 853

Schmid, H. M. 1992, A\&A, 254, 224

Schmid, H. M., Beuzit, J.-L., Feldt, M., et al. 2006a, in Direct Imaging of Exoplanets: Science \& Techniques, eds. C. Aime, \& F. Vakili, IAU Colloq. 200,165

Schmid, H. M., Joos, F., \& Tschan, D. 2006b, A\&A, 452, 657

Schmid, H. M., Joos, F., Buenzli, E., \& Gisler, D. 2011, Icarus, 212, 701

Smith, P. H., Lemmon, M. T., Lorenz, R. D., et al. 1996, Icarus, 119, 336

Sromovsky, L. A. 2005, Icarus, 173, 254

Thuillier, G., Floyd, L., Woods, T. N., et al. 2004, Adv. Space Res., 34, 256

Tomasko, M. G., \& Smith, P. H. 1982, Icarus, 51, 65

Tomasko, M. G., Lemmon, M., Doose, L. R., et al. 1997, in Huygens: Science, Payload and Mission, ed. A. Wilson, ESA SP, 1177, 345

Tomasko, M. G., Buchhauser, D., Bushroe, M., et al. 2002, Space Sci. Rev., 104, 469

Tomasko, M. G., Doose, L., Engel, S., et al. 2008, Planet. Space Sci., 56, 669

Toon, O. B., McKay, C. P., Griffith, C. A., \& Turco, R. P. 1992, Icarus, 95, 24

US Naval Observatory \& Royal Greenwich Observatory 2000, The Astronomical Almanac for the year 2002 (Washington, USA, London, UK: US Government Printing Office (USGPO) and The Stationary Office)

van de Hulst, H. C. 1980, Multiple light scattering, 2 (New York, NY: Academic Press)

Veverka, J. 1973, Icarus, 18, 657

Vinatier, S., Bézard, B., Fouchet, T., et al. 2007, Icarus, 188, 120

West, R. A., Hart, H., Simmons, K. E., et al. 1983, J. Geophys. Res., 88, 8699

Zellner, B. 1973, Icarus, 18, 661 